Florida International University FIU Digital Commons

$11-8-2011$

\title{
An Evaluation of Functional Impairment among Children with Anxiety Disorders
}

Irina Fredericks

Florida International University, ifred001@fiu.edu

DOI: $10.25148 /$ etd.FI11120208

Follow this and additional works at: https://digitalcommons.fiu.edu/etd

\section{Recommended Citation}

Fredericks, Irina, "An Evaluation of Functional Impairment among Children with Anxiety Disorders" (2011). FIU Electronic Theses and Dissertations. 479.

https://digitalcommons.fiu.edu/etd/479

This work is brought to you for free and open access by the University Graduate School at FIU Digital Commons. It has been accepted for inclusion in FIU Electronic Theses and Dissertations by an authorized administrator of FIU Digital Commons. For more information, please contact dcc@fiu.edu. 


\section{FLORIDA INTERNATIONAL UNIVERSITY}

Miami, Florida

\section{AN EVALUATION OF FUNCTIONAL IMPAIRMENT AMONG CHILDREN WITH ANXIETY DISORDERS}

A dissertation submitted in partial fulfillment of the

requirements for the degree of

DOCTOR OF PHILOSOPHY

in

PSYCHOLOGY

by

Irina Fredericks

2011 
To: Dean Kenneth G. Furton

College of Arts and Sciences

This dissertation, written by Irina Fredericks, and entitled An Evaluation of Functional Impairment among Children with Anxiety Disorders, having been approved in respect to style and intellectual content, is referred to you for judgment.

We have read this dissertation and recommend that it be approved.

William M. Kurtines

Maureen C. Kenny

Jeremy Pettit

Wendy K. Silverman, Major Professor

Date of Defense: November 8, 2011

The dissertation of Irina Fredericks is approved.

$\begin{array}{r}\text { Dean Kenneth G. Furton } \\ \text { College of Arts and Sciences } \\ \hline \begin{array}{r}\text { Dean Lakshmi N. Reddi } \\ \text { University Graduate School }\end{array}\end{array}$

Florida International University, 2011 


\section{DEDICATION}

I dedicate this dissertation to my parents, who taught me that I can accomplish anything if I have enough faith and determination. 


\section{ACKNOWLEDGMENTS}

I would like to thank the members of my committee, Drs. Wendy K. Silverman, Jeremy Pettit, William Kurtines, and Maureen C. Kenny for helping me in the conceptualization, writing, and data analysis of my dissertation.

I owe my special thank you to my mentor and major professor, Dr. Wendy K. Silverman. Without her invaluable guidance and feedback, it would not be possible to accomplish this work. It is such an honor and privilege to have Dr. Wendy K. Silverman as my mentor and major professor.

I would like to thank Dr. Arango who had been consistently encouraging and had confidence in my abilities to not only complete this work, but to complete it with excellence.

I would also like to extend my sincere gratitude to everyone at the Child Anxiety and Phobia Program. Especially, I would like to thank Dr. Carla Marin whose sound advice and reassurance aided the writing of this work in a number of ways. I also want to thank Dr. Sandra Williams who was particularly helpful in guiding me through the basics of Mplus. Without their help, the completion of this work would have been very difficult. Finally, I would like to thank my friends and family, especially, my son, Glenn, who supported me when my frustration was high, my confidence was low, and my spirit was weak. Without their unconditional love and inspiration, I would not be able to complete this work. Thank you ever so much. 


\section{ABTRACT OF THE DISSERTATION \\ AN EVALUATION OF FUNCTIONAL IMPAIRMENT AMONG CHILDREN WITH ANXIETY DISORDERS}

by

Irina Fredericks

Florida International University, 2011

Miami, Florida

Professor Wendy K. Silverman, Major Professor

Despite a considerable progress in developing and testing psychosocial treatments to reduce youth anxiety disorders, much remains to learn about the relation between anxiety symptom reduction and change in youth functional impairment. The specific aims of this dissertation thus were to examine: (1) the relation between different levels of anxiety and youth functional impairment ratings; (2) incremental validity of the Children Global Assessment Scale (CGAS); (3) the mediating role of anxiety symptom reduction on youth functional impairment ratings; (4) the directionality of change between anxiety symptom reduction and youth functional impairment; (5) the moderating effects of youth age, sex, and ethnicity on the mediated relation between youth anxiety symptom reduction and change in functional impairment; and (6) an agreement (or lack thereof) between youths and their parents in their views of change in youth functional impairment vis-à-vis anxiety symptom reduction.

The results were analyzed using archival data set acquired from 183 youths and their mothers. Research questions were tested using SPSS and structural equation modeling techniques in Mplus. 
The results supported the efficacy of psychosocial treatments to reduce the severity of youth anxiety symptoms and its associated functional impairment. Moreover, the results revealed that at posttreatment, youths who scored either low or medium on anxiety levels scored significantly lower on impairment, than youths who scored high on anxiety levels. Incremental validity of the CGAS was also revealed across all assessment points and informants in my sample. In addition, the results indicated the mediating role of anxiety symptom reduction with respect to change in youth functional impairment at posttest, regardless of the youth's age, sex, and ethnicity. No significant findings were observed with regard to the bidirectionality and an informant disagreement vis-à-vis the relation between anxiety symptom reduction and change in functional impairment.

The study's main contributions and potential implications on theoretical, empirical, and clinical levels are further discussed. The emphasis is on the need to enhance existing evidence-based treatments and develop innovative treatment models that will not only reduce youth's symptoms (such anxiety) but also evoke genuine and palpable improvements in lives of youths and their families. 


\section{TABLE OF CONTENTS}

CHAPTER

PAGE

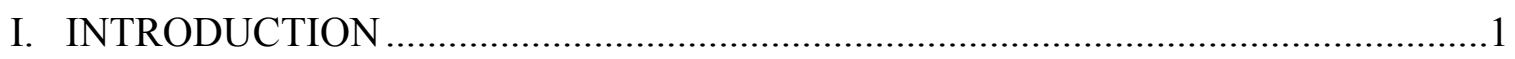

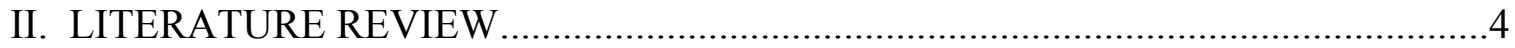

Functional Impairment and Its Special Aspects........................................................4

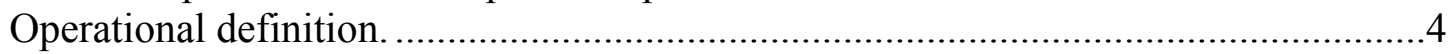

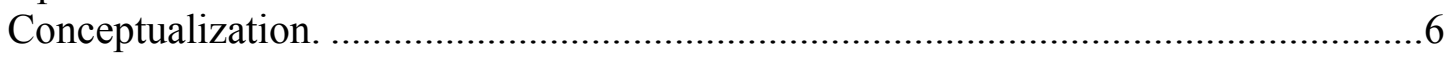

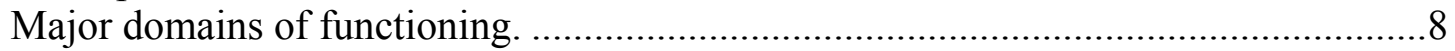

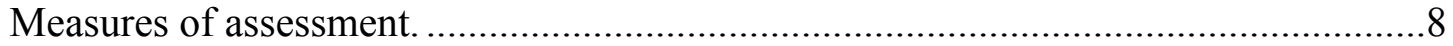

The Evaluation of Functioning in Youth with Anxiety Disorders..................................12

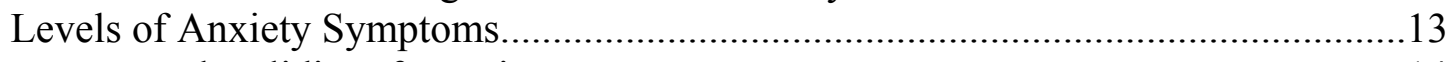

Incremental Validity of Impairment Measures .................................................... 14

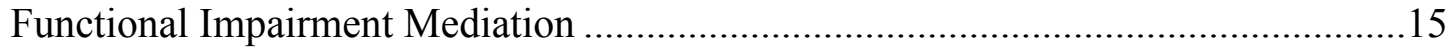

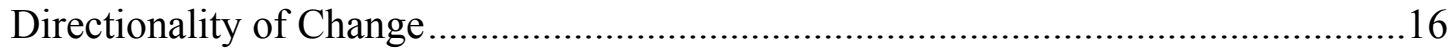

Moderating Roles of Youth Age, Sex, and Ethnicity .......................................... 17

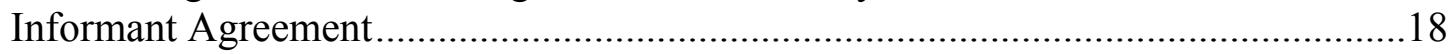

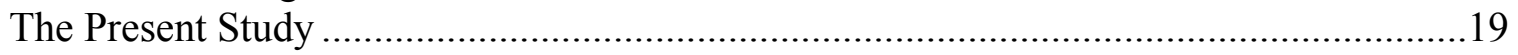

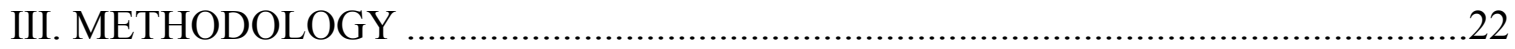

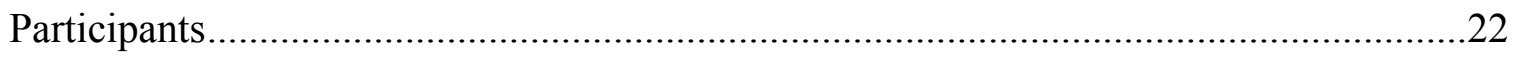

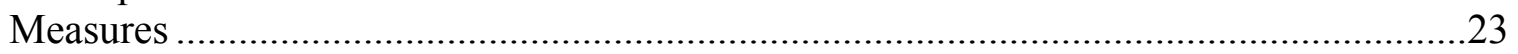

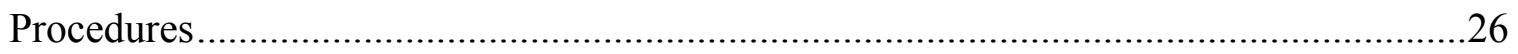

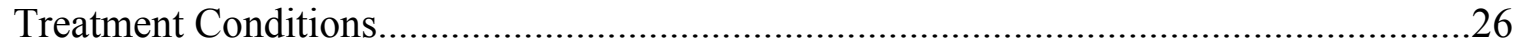

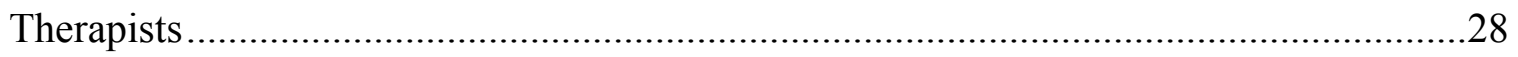

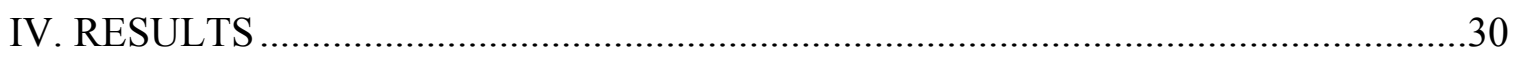

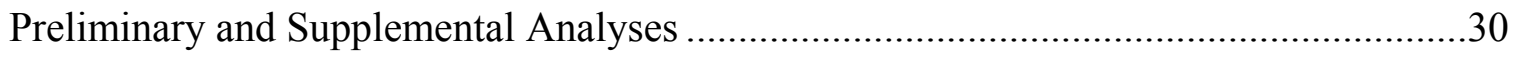

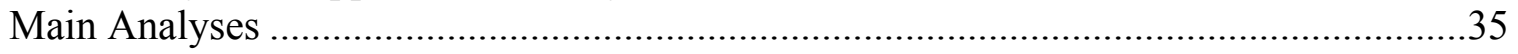

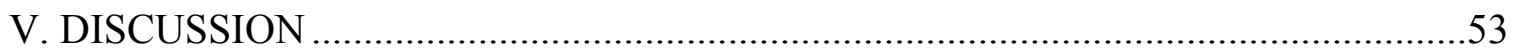

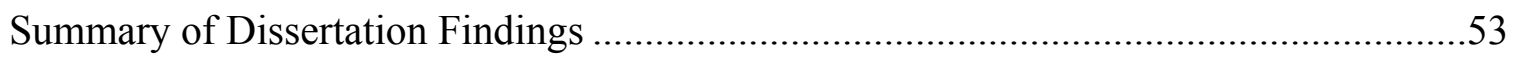

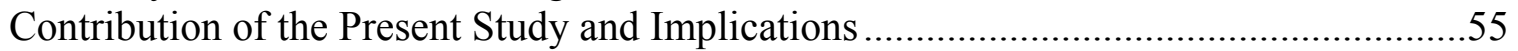

Limitations and Future Research Directions..............................................................61

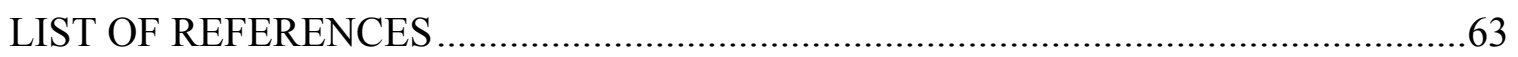

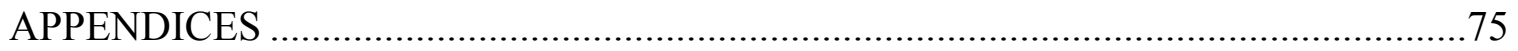

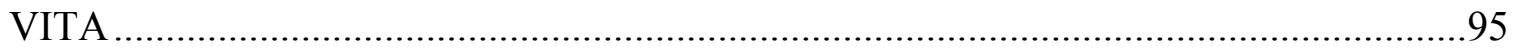




\section{LIST OF TABLES}

TABLE

PAGE

1. Demographic and Diagnostic Information by Treatment Condition .76

2. Mean (Standard Deviations) for Outcome and Mediator Measures .78

3. Mean Differences for Outcome and Youth Completed Measures................................79

4. Mean Differences for Outcome and Parent Completed Measures...............................80

5. Incremental Validity of the CGAS in relation to combined CSR ...............................81

6. Incremental Validity of the CGAS in relation to ADIS/C CSR ................................82

7. Incremental Validity of the CGAS in relation to ADIS/P CSR .83 


\section{LIST OF FIGURES}

FIGURE

PAGE

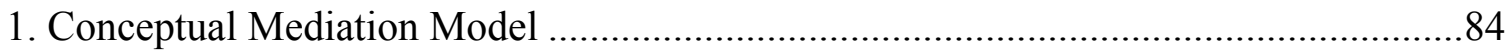

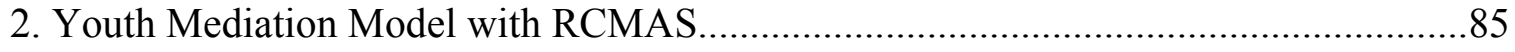

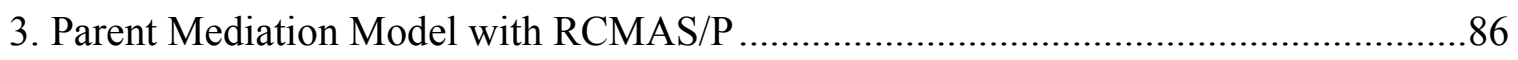

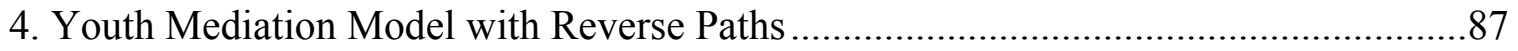

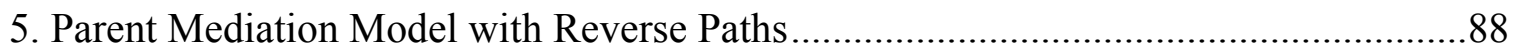

6. Youth Mediation Model with Product Term ...............................................................89

7. Parent Mediation Model with Product Term .................................................................90

8. Youth Mediation Model with ADIS-C CSR ..............................................................91

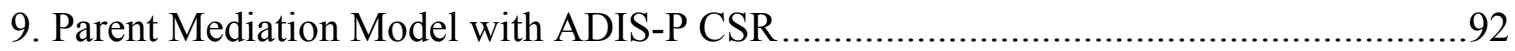




\section{CHAPTER I. \\ INTRODUCTION}

Anxiety disorders represent the single largest mental health problem in childhood and adolescence with the prevalence rate as high as $20.2 \%$ in community and $44.7 \%$ in clinic samples (Costello, Mustillo, Erkanli, Keeler, \& Angold, 2003; Murray, Creswell, \& Cooper, 2009). Anxiety disorders are associated with multiple and significant impairment in psychosocial functioning, including social relations and academic performance (Essau, Conradt, \& Petermann, 2000; Ezpeleta, Keeler, Erkanli, Costello, \& Angold, 2001; Ialongo, Edelsohn, Werthamer-Larsson, Crockett, \& Kellam, 1995; Rapee, Schniering, \& Hudson, 2009). Early-onset anxiety disorders are likely to be chronic if untreated and tend to predispose children to future psychological disorders, such as depression and substance abuse (Cole, Peeke, Martin, Truglio, Serocynski, 1998; Kovacs \& Devlin, 1998; Zahn-Waxler, Klimes-Dougan, \& Slattery, 2000).

In recognition of anxiety disorders' debilitating effects on important areas of functioning, a substantial body of research has evolved over the past two decades evaluating the efficacy of psychosocial treatments for anxiety reduction in children and adolescents (from here on referred to as youth unless specifying a specific developmental period). Silverman, Pina, and Viswesvaran (2008) reviewed and classified the status of evidence-based psychosocial treatments in 32 studies using Chambless et al.'s (1996), Chambless and Hollon's (1998), and Nathan and Gorman's (2002) criteria. A number of treatments were found to meet criteria for "probably efficacious" and "possibly efficacious." All treatments that employed exposure based Cognitive Behavioral Treatment (CBT) were found to be efficacious whether delivered in individual (ICBT), 
parent involvement (PCBT), or group (GCBT) formats (Barrett, 1998; Barrett, Dadds, \& Rapee, 1996; Beidel, Turner, \& Morris, 2000; Kendall, Hudson, Gosch, FlannerySchroeder, \& Suveg, 2008; Marin, 2010; Silverman et al., 1999; Silverman, Kurtines, Jaccard, \& Pina, 2009; Walkup et al., 2008).

Despite the progress made in treatment development and evaluation, much remains to learn about how successful CBT for anxiety disorders impacts youths' daily life functioning. No study has yet to evaluate directly the relation between symptom reduction and changes in functional impairment among youths with anxiety disorders. Statements about how, under what condition, with whom, and in what direction anxiety symptom reduction impacts youth functioning are therefore likely to be based on assumptions rather than empirical data (Kazdin, 1999). Thus, what the quality of the relation is between symptom reduction and change in youth functional impairment is, at this point, essentially an empirically unanswered question.

There is a need for research that moves beyond questions of treatment efficacy to questions evaluating change in functional impairment among youth with anxiety disorders vis-à-vis anxiety symptom reduction. Specifically, the following questions have not been asked in any youth anxiety treatment study and were asked in this dissertation: (1) What is the relation between different levels (i.e., low, medium, and high) of anxiety symptom severity and youth functional impairment ratings as measured by both the Children's Global Assessment Scale (C-GAS; Shaffer et al., 1983) and the Clinician Severity Ratings (CSR; Silverman \& Albano, 1996)? (2) Do functional impairment ratings measured by the CGAS predict impairment above and beyond measures of anxiety severity symptoms, indicating incremental validity of the CGAS? (3) Does the 
impact of psychosocial treatments aimed at reducing youth phobic and anxiety disorders in a PCBT (targeting parenting behaviors and the parent-youth relationship) and a GCBT (targeting youth social skills behaviors and the peer-youth relationship) approach mediate change significantly in youth functional status? (4) What is the directionality of change in the proposed mediated relation between anxiety symptoms and youth functional impairment? (5) Does the proposed mediated relation between anxiety symptoms and youth functional impairment vary as a function of youth age, sex, and ethnicity? (6) Is there an agreement (or lack thereof) between youths and their parents in their views of change in youth impairment vis-à-vis anxiety symptom reduction?

Providing empirically derived answers to these six research questions constituted the specific objectives of this dissertation. Each of the objectives along with the relevant background information is elaborated upon in Chapter II of the dissertation. As will become apparent shortly from the literature review, the present study is the first empirical investigation in the field of youth anxiety treatment to move beyond efficacy by evaluating youth functional impairment vis-à-vis anxiety symptom reduction. 


\section{CHAPTER II.}

\section{LITERATURE REVIEW}

In this chapter, functional impairment is discussed. The focus is first, on its special aspects, including operational definition and conceptualization, major domains of functioning, and measures of assessment. This is followed by a brief discussion on the scarcity of empirical knowledge about the impact of CBT interventions on functional impairment among youth with anxiety disorders. The emphasis is on whether anxiety symptom reduction is a significant mediator of positive change in youth functional status. A summary of the present study's research questions and hypotheses completes Chapter II of this dissertation.

\section{Functional Impairment and Its Special Aspects}

Operational definition. Functional impairment refers to severe problems in daily life functioning as a consequence of psychological symptoms or disorders, leading to the need for mental health services (Diagnostic and Statistical Manual of Mental Disorders, $4^{\text {th }}$ ed. [DSM-IV-TR]; American Psychiatric Association [APA], 2000). In youth psychological research, the construct of adaptive functioning refers to the relative ability of the youth to effectively care for one's self, interact with others, and meet demands of social role performance and role satisfaction in various settings and situations (Üstun \& Chatterji, 1997). High levels of functional impairment predict the likelihood youth will be referred for treatment, more so than psychiatric symptoms alone (Bird, 1999; Kazdin, 1999). Impairment can interfere substantially with or limit youths' functioning in terms of all important life skills (e.g., communication, self-care, social/interpersonal, self- 
direction, academic, leisure, and safety) in family, school, or community settings (APA, 2000; Bird, 1999).

For nearly two decades, the $D S M-I V-T R$ (APA, 2000) taxonomy required the concept of 'mental disorder' to be applied only to individuals who both meet the symptom criteria for a disorder and experience a significant degree of functional impairment associated with the symptoms (APA, 2000). The ascertainment of functional impairment thereby gained relevance, including in clinical research and practice, as well as epidemiological investigations. Impairment in functioning is a criterion for determining clinical diagnoses, treatment efficacy, mental health service eligibility, and psychological disorder prevalence estimates (Bird, 1999; Costello, Egger, Copeland, Erkanli, \& Angold, 2009; Kramer et al., 2004).

Functional impairment is difficult to operationalize and ascertain because it is in part a subjective epiphenomenon that depends on a number of individual factors, such as intelligence, competence, personal characteristics, and communication styles (Bird, 1999). Measuring degree of functional impairment can further be influenced by contextual factors, such as the culture of the environment and society's goals and expectations (Angold et al., 2002). This notion is especially relevant to clinicians and researchers in the USA whose work often involves evaluating individuals from different ethnic groups and cultural backgrounds. An individual's functional status may incorrectly be judged as impaired because of a rater's or clinician's unawareness of the individual's "cultural frame of inference" (APA, 2000, p. xxxiv) for adaptive functioning.

Operationalizing youths' functioning is also complicated by the need to consider developmental factors to evaluate whether the youth's ability to cope with common life 
demands and meet the standards of personal independence is at the level appropriate for his or her age group (Bird, 1999). "Functional impairment" is therefore a multifaceted and multidimensional concept, the operational definition and ascertainment of which requires taking into account various individual and contextual factors interacting in multitude and complex ways (APA, 2000).

Conceptualization. As stated by Kazdin (1999), the concept of functional impairment is closely linked with but "readily distinguishable" (p. 333) from the concepts of personal distress, and the symptoms and severity of a given psychological disorder. Specifically, personal distress represents one's subjective evaluation of levels of stress, discomfort, and emotional pain caused by psychological symptoms (Ezpeleta, Reich, \& Granero, 2009). Functional impairment, in contrast, refers to the consequences that psychological symptoms or disorders have on the youth's performance in all important areas of functioning (Üstun \& Chatterji, 1997). Although personal distress has often been used as a proxy for impairment (i.e., symptoms/disorders that cause functional impairment at home, at school, or with friends also cause high levels of distress), the concepts of functional impairment and personal distress are not interchangeable and thus should be evaluated independently (Ezpeleta et al., 2009).

Compared to symptoms that represent the behavioral feature of a given disorder, and severity that represents the level of seriousness of the disorder, impairment in adaptive functioning represents the disorder's impact on the individual and others (Bird, 1999). Although both severe symptoms and functional impairment are core diagnostic features of all disorders listed in $D S M-I V-T R$ and both are required criteria for diagnoses, they are not invariably related (Kasdin, 1999). 
Distinguishing between the concepts of symptoms and impairment is important because a number of studies have shown there are youths who do not meet $D S M-I V-T R$ criteria for a specific disorder but who have symptoms associated with functional impairment and thus are still in need of mental health services (Angold \& Costello, 1996; Costello \& Shugart, 1992; Wells, Burnam, Rogers, Hays, \& Camp, 1992). For instance, Angold, Costello, Farmer, Burns, and Erkanli (1999) found that youths with subthreshold disorders but who had symptomatic impairment were as disturbed as youths with a diagnosis but without impairment. These findings raise questions about whether the field's focus on developing and evaluating disorders specific treatments, although has moved the field forward, still is limited by its relative inattention to the relation between symptom reduction and improvement in impairment.

As Kazdin (1999) pointed out, clinical significance in treatment outcome research has largely been evaluated by symptom reduction. However, treatments may reduce symptoms to normative levels but not necessarily produce significant changes in functional impairment. It is therefore important to evaluate whether symptom reduction mediates improvements in youth functional status. Empirical knowledge about how (e.g., significantly or not, negatively or positively), under what condition (e.g., severity level, point of development), with whom (e.g., girls versus boys), and in what direction (e.g., symptom to impairment or vice versa) symptom reduction produces change in youth functional impairment can help to improve evidence-based treatments' designs, delivery, and outcomes. Symptom reduction is important but it may not reflect significant and palpable changes in youth's daily functioning (Kazdin, 1999). 
Major domains of functioning. In child and adolescent research, functional impairment has been evaluated along three major domains (Bird, 1999). The first domain is interpersonal relationships. Interpersonal relationships concern both the qualitative and quantitative aspects of youths' relations with peers, family members, and other adults with whom youths interact on a regular basis outside the family (e.g., teachers, church leaders). The second domain is the academic performance. Academic performance concerns the youth's ability to perform in school without undue anxiety and at the level consistent with their potential. The third domain is the capacity to enjoy life. The capacity to enjoy life concerns the youth's utilization of leisure time for self-fulfillment through a wide range of extracurricular activities, interests, or hobbies. Other specific areas that relate to these three domains and, according to Bird (1999), are necessary to evaluate are an age-appropriate cognitive level, verbal skills, and a capacity for self-care.

Measures of assessment. A variety of assessment scales have been developed to assess youth functioning (Hodges \& Gust, 1995). A number of scales are domain specific, evaluating youth functioning in one of the major domains discussed above. For instance, peer sociometric rating scales (e.g., Teacher Assessment of Social Behavior; Cassidy \& Asher, 1992) evaluate youths' functioning with respect to interpersonal relationships. Standardized intelligence and academic achievement tests (e.g., Wechsler Intelligence Scale for Children - fourth edition; Wechsler, 2004) evaluate youths' functioning with respect to their potential for academic performance. Other instruments are multidimensional, assessing youth functioning across the three major domains. For instance, the Child and Adolescent Functional Assessment Scale (CAFAS; Hodges \& Kim, 2000; Hodges \& Wong, 1996) is an interviewer and caregiver rated measure of 
youths' functional impairment with regard to their interpersonal relations, academic performance, and self-fulfillment.

Although these and other domain specific and multidimensional measures are well-validated measures of impairment (Hodges \& Gust, 1995), they have limitations (Fabiano et al., 2009). The use of domain specific scales requires administration of multiple measures to obtain comprehensive assessment of youth functional impairment, limiting their practical utility, for example, in terms of repeated measures. The use of multidimensional measures of functional impairment in youth, such as the CAFAS, is limited by the need to administer an interview, as well as the lack of uniformity across informants (Fabiano et al., 2009).

An alternative way to evaluate youth functioning is by using global measures of impairment. Given that functional impairment refers to overall functioning, global impairment ratings are perhaps the most useful scales to assess youth functional status (Bird, Canino, Rubio-Stipec, \& Ribera, 1987). Global measures conceptualize impairment as one, unified construct of youth's overall functioning across the major domains discussed earlier, providing a single score that represents the amount of impairment that the psychological symptoms or disorders cause the youth. A wide range of scales has been developed to measure global impairment, including Axis V of DSM$I V-T R$ (APA, 2000) and the Columbia Impairment Scale (CIS; Bird et al., 1993). One of the most widely used global measures of youth impairment is the Children's Global Assessment Scale (CGAS; Shaffer et al., 1983).

The CGAS is a clinician rated scale of adaptive functioning derived from the Global Assessment Scale for adults (Endicott, Spitzer, Fleiss, \& Cohen, 1976). The scale 
is divided into 10 deciles that contain references to behavioral descriptors of clinical manifestation of symptoms in terms of their impact on youths' school performance, relationships to others, interests, and hobbies. Based on these descriptors, raters synthesize the knowledge about the youth's psychological and social functioning and assign a single numerical global score of impairment ranging from 100 (highest level of functioning) to 1 (lowest level of functioning).

Numerous psychometric studies have been conducted on the CGAS, demonstrating its utility in discriminating between clinical and nonclinical groups (Bird et al., 1987; Schorre \& Vandvik, 2004; Shaffer et al., 1983; Steinhausen \& Metzke, 2001). Because the CGAS was used in this dissertation, further information on its psychometric properties is provided in the Measures section. Of note is that the CGAS does not provide specific information on impaired areas of youth functioning. Nonetheless, its anchor points within the respective rating decile reflect both symptom severity and interference, adding "a dimension to the definition of disorders that goes beyond categorical diagnostic ratings or symptom-specific scales" (Bird, 1999, p. 218).

In child and adolescent anxiety treatment research, the Clinician Severity Rating scale (CSR; Silverman \& Albano, 1996) is one of the most widely used global measures of youth impairment. The CSR is part of the Anxiety Disorders Interview Schedule for DSM-IV: Child and Parent Versions (ADIS-C/P; Silverman \& Albano, 1996). The ADIS-C/P is a semi-structured interview administered to the youth and parent to assess the presence of anxiety and other psychological disorders. Based on youth and parent informants, the CSR assesses the relative interference of the symptoms reported with respect to youths' school performance, friendships, family relations, and youths' personal 
distress. In addition, the CSR assesses whether youth functioning is at the age-appropriate level, indicating the scale's sensitivity to developmental factors.

The CSR scores range from 0 (Not At All) to 8 (Very, Very Much), with the scores of 4 or higher viewed as definitely interfering and impairing. To derive a combined score of the CSR, an interviewer considers both the youth's and parent's views and adjusts the interference score to the higher of the two CSRs for each endorsed diagnosis. Because the CSR was used in this dissertation, further information on its psychometric properties is provided in the Measures section. Given that a decrease in subjective distress and severity is a measure of clinical improvement (APA, 2000; Ezpeleta et al., 2009), the CSR is useful for assessing treatment efficacy.

The evaluation of youth functional impairment, as assessed by both the CGAS and the combined CSR, is useful. First, it allows for the assessment of youth functional impairment within a developmentally sensitive framework, which accounts for both the domains and degree of interference relative to normative youth development. Second, it provides for an understanding of the degree to which an individual child or adolescent is dysfunctional or impaired. Third, it allows researchers and clinicians to ascertain whether a specific psychosocial treatment produces meaningful and significant changes in lives of youths and their families (Kazdin, 1999). Finally, it allows for the evaluation of incremental validity of the CGAS in relation to the CSR. Incremental validity refers to "the degree to which a measure explains or predicts a phenomenon of interest, relative to other measures" (Haynes \& Lench, 2003, p. 456) and depends on the goal of the assessment, whether it is to improve diagnostic efficacy or evaluate treatment outcome (Johnston \& Murray, 2003). Evaluating incremental validity of the CGAS can help to 
ascertain its utility in predicting impairment above and beyond measures of anxiety severity symptoms.

\section{The Evaluation of Functioning in Youth with Anxiety Disorders}

The presence of functional impairment is an explicit component of all psychological disorders listed in $D S M-I V-T R$, including youth phobic and anxiety disorders. In fact, the symptoms of an anxiety disorder are only valid indexes if they cause persistent, pervasive, and significant interference or impairment in social, academic, or other important areas of functioning (APA, 2000). Research suggests that the prevalence rate of youth anxiety disorders, especially specific phobia, is the most affected by including an evaluation of functional impairment to make a diagnosis, more so than that of all other diagnoses (Costello et al., 2009). Thus, when evaluating anxiety treatment outcome, it is important to examine its impact on not only the symptoms of a given anxiety disorder but also level of functional impairment associated with the symptoms (Kazdin, 1999).

As stated earlier, the past two decades have witnessed a considerable progress in developing and testing psychosocial treatments to reduce youth anxiety disorders. As a result, there now exists a large body of research supporting the efficacy of CBT for treating anxiety disorders in youth (e.g., Silverman et al., 2008). Existing research evidence supporting the efficacy of CBT to reduce youth anxiety symptoms however does not necessarily mean that $\mathrm{CBT}$ outcome (i.e., symptom reduction) is efficacious to reduce impairment in youth's functioning- it may or may not (Kazdin, 1999). It is important to demonstrate this empirically. The issue of the field's relative inattention to change in youth functional status vis-à-vis successful treatment outcomes with respect to 
how, under what condition, with whom, and in what direction the change occurs thus comprised the main goal of this dissertation. Its specific aims are elaborated upon in more detail in the following sections.

\section{Levels of Anxiety Symptoms}

Not one study has yet investigated the relation between different levels (i.e., low, medium, high) of youth anxiety symptoms and functional impairment. Thus, extending past outcome research by evaluating directly whether low, medium, and high level of anxiety symptoms differentially relate to youth functional impairment was the first objective of this dissertation. The evaluation of the linkages between youth levels of anxiety symptom and functional impairment ratings is important because it can help to address the issue of metrics arbitrariness in psychological research and practice. As stated in Blanton and Jaccard (2006), "metric" is a rating a person gives to items on psychological measures. A metric is arbitrary when it is unknown how a change in scores translates into the change in the person's real world experiences. For instance, a child receives a score of 15 on an anxiety measure at pretreatment and a score of 5 at posttreatment. The meaning of the anxiety score change is arbitrary because it does not convey how it translates into the change in the child's real world experiences. One strategy to reduce the arbitrariness of metrics is norming approach (Blanton \& Jaccard, 2006). This approach involves grouping anxiety symptom severity scores in terms of magnitude (i.e., low, medium, or high) and linking them to observable and meaningful changes in the outcome of interest - namely, youths' functional status across major domains of functioning discussed earlier. Although not sufficient to generate nonarbitrary 
metrics, the norming procedure can help to address the issue of metric arbitrariness (Blanton \& Jaccard, 2006).

Evaluating the linkages between different levels of anxiety and functional impairment can also help to identify individuals whose mental health is a cause for concern and who are considered to be in need of mental health services. This knowledge is important because it can (1) help clinical researchers to prioritize interventions, (2) better understand the phenomenology of disorders in youth, (3) refine diagnostic criteria, and (3) guide community-based mental health administrators in their decision making with respect to resource allocation for youths and their families seeking services in real world clinical settings (Bird, 1999).

\section{Incremental Validity of the CGAS}

As discussed earlier, the CGAS and the CSR are the most useful global measures of youth functional impairment in youth psychological research. Not one study however has yet evaluated predictive or incremental validity of the functional impairment measures -- particularly, whether CGAS ratings account for unique and substantial variance in CSR scores, independent of measures of anxiety symptom severity. Thus, extending past outcome research by assessing incremental validity of the CGAS to evaluate its predictive value of impairment above and beyond information already available (e.g., measures of anxiety symptom reduction) was the second objective of this dissertation.

Evaluating empirically whether CGAS ratings predict impairment above and beyond anxiety symptom measures is important. It can help to examine the efficiency of assessment protocols, justify the use of exiting assessment measures, and hence to 
ascertain that psychological services are carried out in a way that is maximally beneficial to clients (APA, 2002; Johnston \& Murray, 2003). Addressing incremental validity is also useful in research involving multiple informants (e.g., clinicians, youths, parents) because it helps to evaluate the contribution of each informant to the outcome of interest (Haynes \& Lench, 2003). Taken together, evaluation of incremental validity is important because it can provide a fine-grained assessment of treatment outcome that pays due attention to improvements in functional impairment following treatment, associated with but assessed independently from the symptoms.

\section{Functional Impairment Mediation}

No study in the field of treatment outcome has yet evaluated the mediating role of the impact of anxiety symptom reduction on youth functional impairment. Extending existing outcome research by providing empirical knowledge about whether anxiety symptom reduction mediates significantly change in youth functional impairment was the third objective of the present study.

There are several reasons why empirical evaluation of youth functional impairment mediation by positive treatment outcomes is important. First, evaluating the impact of anxiety symptom reduction on youth functional impairment may advance theoretical understanding about the processes by which interventions produce positive changes in youths' functional status. This understanding is important given scarcity of empirical knowledge about issues of mechanisms and theories of change in child psychotherapy (Jensen, et al., 1999; Kazdin, 2001, 2007). Second, knowledge of how change in youth functional impairment is achieved is important for practical reasons because it can help to identify mediating variables that trigger this critical change. Once 
this specific variable is identified, it can be included as the key components in the therapeutic processes to enhance the design and delivery of existing treatment strategies so that they will render maximally efficient (i.e., effective) interventions (Kazdin, 2001).

Third, revealing the key components that produce meaningful changes in youth functional impairment can help to move the field beyond the current research of treatment outcome toward research of innovative treatment models that will not only reduce youth's symptoms (such as anxiety) but also evoke practical and palpable improvements in lives of youths and their families (La Greca, Silverman, \& Lochman, 2009). Fourth, advancing knowledge about the mechanisms underlying the change in youth functioning holds public health significance. That is, using empirical knowledge about what leads to improvements in youth functional impairment, and what does not will help to develop more efficient and effective psychosocial treatments to reduce youth psychopathology (Berman, Weems, Silverman, \& Kurtines, 2000; Kazdin, 2008).

\section{Directionality of Change}

Also important but has not been explored is the directionality of change in the relation between anxiety symptom reduction and improvement in youth functional impairment. Bidirectional causal relations and processes frequently appear in the psychological theory (e.g., developmental systems theory approach; Gottlieb, 2007). Nonetheless, empirical investigation of bidirectional influence between variables is not frequently pursued in psychological research, which may in part be due to statistical and methodological issues (e.g., underidentification and insolubility) inherent in the fitting of bidirectional influence models (Bollen, 1989; Kenny, 1979). In the field of treatment outcome, for instance, Silverman et al.'s (2009) randomized controlled trial is the only 
study to examine and find some evidence for the bidirectionality of change in the relation between youth anxiety and parent variables (i.e., youth to parent, parent to youth, or bidirectionally).

Extending past outcome research by evaluating empirically whether the directionality of change to the relation between symptom reduction and youth functional impairment is bidirectional was the fourth objective of this dissertation. This knowledge is important given that many models currently evaluated in outcome research are based on the assumption of unidirectional causality and thus are somewhat limited in their theoretical and practical potential (Martner \& Haase, 2006). Finding that the dynamics of change may not only flow from symptom reduction to impairment improvement but also from impairment improvement to symptom reduction can help to begin filling the knowledge gap about the complex nature of the proposed mediated relation between anxiety symptom reduction and youth functional impairment.

\section{Moderating Roles of Youth Age, Sex, and Ethnicity}

Also not examined is whether there are the moderating roles of youth age, sex, and ethnicity with respect to the proposed mediated relation between anxiety symptom reduction and youth functional impairment. Extending the treatment outcome research by addressing this issue directly was the fifth objective of this dissertation. Determining whether the proposed mediated relation between positive treatment outcomes and youth's functional status varies as a function of youth age is important because it can inform the field about differences in the developmental expression of functional impairment (Weems \& Costa, 2005). Examining a moderating role of youth sex can help to expand the conceptual understanding about protective or risk factors (Ginsburg \& Silverman, 2000) 
that can strengthen or weaken, respectively, the impact of treatment gains on youth functional impairment. Examining whether and how youth ethnicity moderates the proposed functional impairment mediated by positive treatment outcome also may assist in formulating and implementing psychosocial interventions sensitive to issues that may arise in working in contexts of cultural diversity (Kurtines \& Szapocznik, 1996).

Taken together, examining the moderating role of youth age, sex, and ethnicity with respect to the effect of successful treatments on youth functional status may assist improving the quality and clinical relevance of evidence-based treatment procedures and outcomes because it can help clinicians to develop treatment plans that meet the needs of a particular child. Because of the lack of past research to guide specific hypotheses with respect to these background variables coupled with the power requirements needed for a full and complete evaluation of these variables as potential moderators, no formal hypotheses about these specific variables and their moderating roles were included in this study.

\section{Informant Agreement}

No study has yet to evaluate an agreement (or lack thereof) between youths and their parents in their view of youth functional impairment vis-à-vis anxiety reduction. Past research suggests that in general, youths' and parents' reports on the association between reported symptom scores and associated impairment differ, irrespective of diagnostic type (Jensen et al., 1999; Wille, Bettge, Wittchen, \& Ravens-Sieberer, 2008). Adding to existing research by evaluating whether youths and their parents disagree in their view of change in impairment vis-à-vis anxiety symptom reduction was the sixth and final objective of the present study. 
Empirical knowledge about informant agreement vis-à-vis the symptomimpairment relation is important. Specifically, as discussed earlier, impairment in youth functioning is difficult to ascertain because it is in part a subjective epiphenomenon (Bird, 1999). Obtaining and comparing empirical information from multiple informants (e.g., youths and their parents) can assist in better understanding how changes in youth functional impairment vis-à-vis anxiety symptom reduction manifest. Given that anxiety, especially generalized worry, is a psychological disturbance that is largely internally experienced, empirically comparing youths and parents' view of youth functional impairment vis-à-vis anxiety reduction may also assist in establishing accurate diagnoses, necessary for maximizing treatment gains. Moreover, using reports from multiple informants about their view of youth functional status vis-à-vis anxiety reduction can help to gain comprehensive information about the magnitude of impairment in youths' functioning, which is crucial for planning, monitoring, and evaluating evidence-based treatments for anxiety disorders in youth (Kramer et al., 2004).

\section{The Present Study}

The specific aims of this dissertation were to evaluate the following research questions and related hypotheses:

1. What is the relation between the low, medium, and high levels of anxiety at posttreatment, as measured by the youth and parent rated Children's Manifest Anxiety Scale - Revised (RCMAS and RCMAS/P; Reynolds \& Richmond, 1978) and Multidimensional Anxiety Scale for Children (MASC; March, Parker, Sullivan, Stallings, \& Connors, 1997), and youth functional impairment ratings, as measured 
by both CGAS (Shaffer et al., 1983) and the combined CSR (Silverman \& Albano, 1996) at posttreatment and 12-month follow up?

Hypothesis 1: The youths reporting the low and/or medium anxiety level(s) at posttreatment will score significantly higher on the CGAS and lower on the CSR at posttreatment and 12-month follow up, compared to those reporting high anxiety levels.

2. Do functional impairment ratings measured by the CGAS predict impairment above and beyond measures of anxiety severity symptoms, indicating incremental validity of the CGAS?

Hypothesis 2. The functional impairment measured by the CGAS ratings will account for unique variance in the combined CSR, as well as youth reports based (i.e. ADIS-C CSR), and parent reports based (i.e., ADIS-P CSR), above and beyond the prediction of impaired functioning from anxiety symptoms alone at posttreatment and 12-month follow up, revealing incremental validity of the CGAS.

3. Are reductions in youth anxiety symptoms, measured by youth and parent rated RCMAS and MASC at posttreatment, significant mediators of change in youth functional impairment, measured by the CGAS and CSR at posttreatment and 12month follow up?

Hypothesis 3 . Anxiety symptom reduction, measured by youth and parent rated RCMAS and MASC at posttreatment, will significant mediate change in youth functional impairment measured by the CGAS and the combined CSR at posttreatment and follow up. 
4. What is the directionality of change in the relation between anxiety symptom reduction and youth functional impairment?

Hypothesis 4 . The proposed mediating relation between anxiety symptom reduction and levels of functional impairment will be bidirectional, indicating that as anxiety symptoms decrease, youth functional impairment ratings decrease as well, and vice versa.

5. What is the role of youth's age, sex, and ethnicity, respectively, on the proposed youth functional impairment mediation by anxiety symptoms reduction at posttreatment and 12-month follow up?

Due to the lack of research with regard to the respective moderating effects of youth age, sex, and ethnicity on the mediated relation between symptom reduction and functional impairment, no hypotheses for this particular research question were formulated. The proposed moderator analyses were performed for the exploratory purposes only.

6. Is there an agreement between youths and their parents in their view of youth functional impairment vis-à-vis anxiety reduction?

Hypothesis 5. There will be a significant difference between youths' and parents' ratings of youth impairment as measured by the ADIS-C and ADIS-P CSRs at posttreatment and 12-month follow up, suggesting a lack of informant agreement in their views of youth functional impairment vis-à-vis anxiety symptom reduction. 


\section{CHAPTER III.}

\section{METHODOLOGY}

\section{Participants}

The present study consists of analysis of an archival data set obtained from 183 youths (ages 6 to 16 years; $M=9.72 ; S D=2.21$ ) and their mothers who presented to the Child Anxiety and Phobia Program (CAPP) at Florida International University (FIU). The CAPP is a research clinic specializing in youth anxiety disorders and its related difficulties. All participants were referred to the CAPP by pediatricians, school psychologists, or other mental health professionals due to difficulties with excessive anxiety. The participants' age range in this current study is comparable with the age range of past randomized clinical trials (Barrett et al., 1998; Kendall, 1994). The data analyzed in the present study only included subjects that completed the treatment.

Sociodemographic information of the study's participants is provided in Table 1. As shown, $75 \%$ of the participants were Hispanic/Latino Americans, and $54 \%$ were boys. The youths' age range of 6 to 16 years reflect the modal age range of the age of onset of separation anxiety disorder (SAD), social phobia (SOP), specific phobia, (SP), and generalized anxiety disorders (GAD) in the population and is reflective of the CAPP's referral patterns.

The study's inclusion criteria for the youth participants were: (a) primary diagnoses of $D S M-I V-T R$ anxiety disorder of SAD, SOP, SP, and GAD, reviewed and confirmed at a staff conference directed by Dr. Silverman following the administration of the ADIS-C/P (Silverman \& Albano, 1996); (b) a mean score of 4 or greater on the CSR (Silverman \& Albano, 1996; see Measures); (c) discontinuation of all other youth 
psychosocial treatment upon review with the program's clinic staff and the service provider; (d) discontinuation of certain psychopharmacological agents that were viewed as confounding the study, upon review with the program's psychiatric consultant and under medical supervision; (e) between 6 and 16 years of age; (f) mothers must agree to participate in their youth's treatment; and (g) agree to be randomized into either a parent involvement (PCBT) or a group (GCBT) treatment condition.

The study's exclusion criteria for the youth participants were: (a) primary diagnoses of any $D S M-I V-T R$ anxiety disorders other than SAD, SOP, SP, and GAD; or (b) refusal to discontinue a psychosocial treatment or psychopharmacological agents as per study's protocol and as per medical supervision; or (c) youth and/or their mothers' diagnoses (e.g., primary, secondary, tertiary) of Pervasive Developmental Disorders, Mental Retardation, Organic Mental Disorders, Schizophrenia or Other Psychotic Disorders; and/or (d) youth and/or their mothers' demonstration of a high likelihood of hurting themselves or others.

\section{Measures}

Functional impairment measures. Children's Global Assessment Scale (CGAS; Shaffer et al., 1983). The CGAS is a clinician rated scale designed to reflect levels of functioning in youth aged 4-16 years. As previously discussed, the scale's scores range from 100 (the highest level of functioning) to 1(the lowest level of functioning), with scores of 67 or higher reflecting functioning within the normal range (Bird et al., 1987). Past research evaluating psychometric characteristics of the CGAS has yielded an interrater reliability coefficient of .66 (intraclass correlation), revealing the scale's considerable strength in providing a global measure of youth functional impairment (Bird 
et al., 1987; Shaffer et al., 1983). As in past research, CGAS ratings were derived during case presentation meetings headed by Dr. Silverman.

Clinician Severity Rating (CSR; Silverman \& Albano, 1996). The CSR is an interviewer/clinician rated scale that was designed to assess the degree of interference associated with the symptoms of the major $D S M-I V-T R$ disorders, such as anxiety, mood, and externalizing disorders, experienced by school-age youth. The CSR is a part of the ADIS - C/P (Silverman \& Albano, 1996), which in addition to producing DSM-IV-TR diagnoses, assigns the CSRs ranging from 0 (Not At All) to 8 (Very, Very Much) for each endorsed diagnosis. The CSR of 4 or higher is viewed as definitely interfering and impairing, and those less than 4 are viewed as subthreshold. To derive a combined score of the CSR, an interviewer considers both the youth and parent's views and adjusts the interference score to the higher of the two CSRs for each endorsed diagnosis. Research studies with the ADIS-C/P revealed its solid psychometric properties. Inter-rater agreement for the ADIS-C/P on the pre-treatment clinician rating scale using the interviewer-observer paradigm has been found to be .74 (Silverman, Cerny, \& Nelles, 1988); .89 for test-retest for the ADIS-C, .87 for the ADIS-P, and .88 for the composite rating (Silverman \& Eisen, 1992). The CSR scores were reviewed and confirmed at a case presentation meetings headed by Dr. Silverman following the administration of ADIS-C/P. The CSR scores were derived prior to obtaining the CGAS ratings.

Anxiety symptom measures. Multidimensional Anxiety Scale for Children (MASC; March et al., 1997). The MASC is a 39-item youth self-rated scale designed to assess youth anxiety disorders. Each scale's item is rated via a 4-point Likert scale. The ratings of the scale's 39 items are summed to yield a Total score of anxiety. The MASC 
is increasingly being used in youth anxiety research as it provides for a more comprehensive and robust assessment of childhood anxiety and its disorders. The MASC's test-retest reliability using a 3-week and 3-month interval is satisfactory to excellent (intra-class correlation coefficients of .93 and .87, respectively) (Baldwin \& Dadds, 2007; March \& Sullivan, 1999). The MASC's convergent and divergent validity is also adequate. Shared variance was highest for scales sampling symptom domains of anxiety, intermediate for depression, and lowest for externalizing symptoms (March et al., 1997).

Children's Manifest Anxiety Scale - Revised (RCMAS; Reynolds \& Richmond, 1978). RCMAS is a 37 -item youth self-rated scale designed to assess youth anxiety symptoms. A Total Anxiety score is computed based on 28 items, which are divided into three anxiety subscales: (1) Physiological Anxiety (i.e., somatic manifestations of anxiety, such as sleep difficulties, nausea, and fatigue); (2) Worry/Oversensitivity (i.e., obsessive concerns about a variety of things of a vague and ill-defined nature, as well as fears about being hurt or emotionally isolated); and (3) Social Concerns/Concentration (i.e., distracting thoughts and fears of a social or interpersonal nature). Each item is rated either Yes or No and scored 1 or 0 , respectively. Because scores are derived from affirmative responses, a high score indicates a high level of anxiety or lie on that subscale. The RCMAS is the most widely used self-rating scale in the youth anxiety treatment research literature (see review by Silverman \& Ollendick, 2005). Pela and Reynolds (1982) reported a 3-week test-retest reliability of .98 for the Total Anxiety scale. Significant correlations have been found between the Total Anxiety scale, trait 
anxiety, and fear ( $r$ s $=.63$ to .88$)$ (Ollendick, 1983). The alpha coefficient in the present sample was .84.

Revised Children's Manifest Anxiety Scale, Parent Version (RCMAS/P; Reynolds \& Richmond, 1978). In RCMAS/P, the wording of RCMAS items was changed from "I..." to "My child..." as done in past research (e.g., Kendall, 1994; Silverman et al., 1999). Each item was rated either Yes or No and scored 1 or 0, respectively. Twenty-eight items are summed to yield a Total Anxiety score. As in the original version of the scale, RCMAS/P contains three anxiety subscales: Physiological Anxiety, Worry/Oversensitivity, and Social Concerns/Concentration. The alpha coefficient in the present sample was .78 .

\section{Procedures}

Assessment interviews and questionnaires were administered after parental informed consent/child assent was obtained. All assessment interviews and questionnaires were generally completed in one session and conducted by doctoral level psychology graduate students. All measures were completed at pretreatment, posttreatment, and 12-month follow up assessment points. Families who met the study's inclusion criteria were invited back to the clinic and informed consent/assent was obtained for their participation in the study.

\section{Treatment Conditions}

As done in previous research (Flannery-Schroeder \& Kendall, 2000; Silverman et al., 1999), participating youth were randomly assigned to either the PCBT or GCBT in blocks of seven. The initiation of the random assignment process to either treatment condition (PCBT or GCBT) was determined by the toss of a coin. In GCBT condition, 
assignment to treatment in blocks of seven was used to avoid delay in the formation of groups. To standardize the content of each treatment session, treatment manuals for PCBT and GCBT were used in the study. In the manuals, therapists were reminded of the main goals, tasks, and critical issues for each session, homework assignments, treatment schedules, etc. (to view a detailed outline of the basic core program as presented to participants, see Marin, 2010). Therapists were advised however to take into account the developmental needs of the youth and proceed accordingly with the treatment protocol.

Given the high proportion of Hispanic families in the sample, $8 \%$ of the treatments were delivered in a bilingual format (English and Spanish) by the request of the Spanish-only speaking parents participating in PCBT. There were no statistically significant differences on any of the primary outcome variables as a result of treatment language in PCBT. All group treatments were delivered in English. The total number of sessions in both PCBT and GCBT was 12 to 14 sessions.

In PCBT, the youth and parents met with the therapist on a weekly basis for a total of 60 minutes. The basic core of this specific CBT format involved not only graded exposures to anxiety provoking situations and training youths in using cognitive and behavioral strategies, but also promoted parents' reinforcement/support for their child's successful handling of his/her avoidant behaviors and improving parenting skills and the parent-child relationship. To target "parenting skills" and "parent-child relationships," the PCBT involved the following main therapeutic components: (1) training parents in managing their children's anxiety and avoidant behaviors, (2) having parents set appropriate contingencies for successful child non-avoidance, (3) and helping parents to 
enhance their acceptance/warmth toward their children with improved communication and problem-solving skills.

In GCBT, the youth met in the group with the therapist on a weekly basis for a total of 60 minutes. The basic core of this particular program involved not only graded child exposures, but also promoted peer reinforcement/support for youth's successful handling of their avoidant behaviors and improving child social skills and peer-child relationships. To target "child social skills" and "peer-child relationships," the GCBT involved the following main therapeutic components: (1) training youth in being more helpful and positive toward other children in the group in the context of fear/anxiety reduction as well as other behaviors, (2) having youth accept the help and support of other group members in role-plays, and (3) training youth in social skills (e.g., compliment giving, conversational skills). As done in previous research (Barrett, 1998; Flannery-Schroeder \& Kendall, 2001), at the start of the treatment program, the middle, and the end, the parents of the youth who have been assigned to GCBT had three brief group meetings (about 30 minutes) with each group therapist to be informed about the program and the youth's tasks and activities.

\section{Therapists}

Because the two conditions require similar therapeutic skill levels, therapists were crossed between conditions as recommended by Kazdin (1994). Crossing therapists with treatment condition allows for an analysis of the therapist variance (i.e., the portion of patient treatment gains attributed to the therapists) that can be separated from the treatment variance (i.e., the portion of patient treatment gains attributed to treatment conditions). In this study, the therapists were eight doctoral level psychology graduate 
students who received training in the proper administration of the treatments by Dr. Silverman. The training of therapists included the following: Therapists first familiarized themselves with the treatment protocols. Particular emphasis was placed on highlighting both the overlap between the conditions (e.g., youth exposure) and the important distinctions between the two conditions (e.g., parenting behaviors versus social skill behaviors). Dr. Silverman provided both didactic and clinical training via extensive role-playing of the treatment's procedures. There were no statistically significant differences between any of the therapists on any of the primary outcome variables.

During the course of the dissertation, Dr. Silverman conducted weekly supervision meetings with the therapists to prepare for upcoming sessions and process sessions that were just completed. This included the review of the therapists' treatment notes, listening to a random selection of therapists' session tapes, and providing ongoing feedback via instructions, recommendations, and role-plays. 


\section{CHAPTER IV.}

\section{RESULTS}

\section{Preliminary and Supplemental Analyses}

Outlier. Analyses were pursued using both non-model based and model-based methods. With respect to the former, multivariate outliers were identified by calculating leverage indices for each respondent. A leverage score four times greater than the mean leverage was defined as an outlier. There were no outliers found in the data using this approach. A model-based outlier analysis was also conducted. This involved regressing a randomly selected indicator for each endogenous variable onto an indicator for variables of which the endogenous variable was assumed to be a linear function. The regression analysis uses ordinary least squares (OLS) regression in a limited information estimation framework. Standardized $d f$ beta estimates were then examined for the predictors and intercepts for each case. Outliers were defined as the absolute value of standardized $d f$ beta scores in the excess of 1.0. No outliers were evident in the data using this approach.

Non-Normality. Univariate indices of skewness and kurtosis were examined to determine whether the absolute value of any of these indices is greater than 2.0. Nonnormality was evident in several variables in this study. The decision was thus to pursue structural equation modeling (SEM) analyses in Mplus 6 (Muthén \& Muthén, 2007) by using an estimator (MLR) robust to violations of normality based on the Huber-White algorithm (Huber, 1967; White, 1980).

Missing data. In the analysis of missing data, it was first determined whether there was a systematic bias in the patterning of missing data. This involved computing a dummy variable reflecting the presence or absence of missing data for a given measure in 
the data. This dummy variable was then correlated with all other variables in the model as well as an array of demographic variables. No significant correlations were observed, indicating that there was no evidence of bias resulting from missing data.

Two types of analyses relevant to the missing data were pursed. For the analyses using the Statistical Package for the Social Sciences (SPSS), missing data were accommodated by using the multiple imputation strategy (Little \& Rubin, 1989). For the analyses using the SEM framework in Mplus 6, the full information maximum likelihood (FIML) missing data methodology (Wothke, 2000) was employed.

Statistical power and sample size considerations. Structural equation modeling requires taking into account statistical power, issues of the stability of the covariance matrix, and the use of asymptotic theory. With regard to statistical power, however, it is difficult to evaluate the power associated with specific path coefficients in complex SEM models because of the large number of assumptions about population parameters that must be made. With regard to the asymptotic theory and covariance stability, simulation studies tend to suggest that sample sizes of 100 to 125 or larger often yield adequate results given that reasonably reliable measures are used (reliabilities greater than 0.65 ) and with a reasonable number of indicators per latent variable (Jackson, 2003; Jaccard \& Wan, 1996). The sample size of 183 participants along with psychometrically sound measures used in the present study exceeds this standard.

Structural equation modeling. To explore the proposed models in this study, the data were analyzed using SEM in Mplus 6. SEM is a powerful and flexible tool that, besides assessing the directional and non-directional relationships among observed and unobserved variables, recognizes the imperfect nature of the measurement and thus 
explicitly specifies error terms for all the measurements in a model (Byrne, 2001). Such specifications are important because traditional multivariate methods like regression assume variables in the analysis are perfectly reliable or free of measurement error. Additionally, SEM provides flexibility, more so than alternative approaches (e.g., regression analysis), because it does not require that a variable be either a cause or an effect of another variable. As a result, SEM allows to test for the presence (or lack thereof) a bidirectional causality among variables of interest. Finally, SEM affords formal significance testing of competing conceptual models to identify those models that are the most appropriate given the data (Byrne, 2001).

Fit indices. Model fit was evaluated with the comparative fit index (CFI) of values $\geq 0.95$, the root-mean-square error of approximation (RMSEA) of values $\leq 0.08$, and the $p$ value for close fit test of values $\geq 0.05$ representing acceptable fit (Bentler, 1990; Brown \& Cudeck, 1993). More focused fit indices were also examined with modification indices of values $\leq 3.83$ and standardized residual values of $\leq 1.96$ representing acceptable fit.

Covariates. In all model tests using SEM in Mplus 6, youth sex, age, and ethnicity were used as covariates. Paths were included from each of these variables to the mediators and outcome variables. The scores of the pretreatment measures of the CGAS and CSR were used as covariates as well (Rausch, Maxwell, \& Kelly, 2003). Correlations were also estimated between primary and secondary outcome measures (i.e., CGAS and CSR) at the posttreatment and follow up in both youth and parent models. To avoid clutter, Figures 1 through 9 exclude the pretreatment scores of the outcome variables, 
youth sex, age, and ethnicity, as well as the correlations among all exogenous and outcome variables; however, all were included in all model tests.

Clustering effects. The GCBT condition consisted of 19 separate treatment groups of youths in blocks of seven. As a result, the model was adjusted for potential clustering effects (19 clusters). Because each case in PCBT is independent from each other, youth participants in the PCBT condition were also coded as separate clusters (100 clusters), for a total of 119 clusters. Given that traditional OLS regression approaches assume independence of observations, intra-class correlation (ICC) coefficients were calculated to examine the degree of non-independence of observations as a result of the clustering of participants in GCBT. This calculation is important because as the ICC increases, the amount of independent information from the data decreases, inflating the Type I error rate of an analysis that ignores this correlation (Blair, Higgins, Topping, \& Mortimer, 1983). If clustering is not of concern, then ICC's should be zero or near zero. Calculation of ICC's revealed coefficients greater than .05 , which was judged to be large enough to adjust for clustering effects. To account for clustering attributable to GCBT in all analyses in this study, the decision was to pursue the algorithms in Mplus 6.

Tables 2 presents means and standard deviations for youth functional impairment and anxiety symptom measures for youth and parent completed measures. As shown in Table 2, multiple measures of anxiety (e.g., RCMAS, RCMAS/P) and functional impairment (e.g., CGAS) obtained from multiple sources (e.g., youth, parent, and clinicians, respectively) were used in this study. Using SPSS, a series of supplementary analyses were conducted to examine the intercorrelations among all the measures within each category (e.g., the category of outcome measure). The correlation between the 
parents' ratings of youth anxiety and the youths' self-ratings of anxiety as measured by respective versions of RCMAS was $.15(p=.038)$ at the pretest and $.29(p<.001)$ at the immediate posttest. The correlation between the youths' self-ratings of anxiety as measured by RCMAS and MASC was .52 at the pretest and .62 at posttest, with both being statistically significant $(p<.001)$. The correlation between the youths' self-ratings of anxiety as measured by MASC and the parents' ratings of youth anxiety as measured by RCMAS/P was .10 at pretest and .18 at posttest, with only the latter being statistically significant $(p=.018)$. Given these low correlations, youth and parent ratings of anxiety on the respective versions of the RCMAS and MASC at pre and posttreatment assessments were treated as separate measures of youth anxiety symptoms in all model tests.

Likewise, the correlation between the clinicians and the youths' and parents' ratings of functional impairment as measured by the CGAS and the CSR combined, respectively, was -.59 at posttest and -.47 at follow up, with both being statistically significant $(p<.001)$. Given these moderate correlations, the CGAS and the combined CSR ratings were treated as separate indicators of youth functional impairment.

In addition, a series of analyses were conducted to evaluate whether the scores on RCMAS subscales (i.e., physiological anxiety, worry/oversensitivity, and social concerns/concentration) differentially contribute to youth functional impairment ratings. Youth and parent models pertaining to each subscale were tested separately using the SEM framework in Mplus 6. Global and focused fit indices indicated good data fit for all models. Path estimates were examined, and informal comparisons of path estimates of the models were conducted. No theoretically meaningful differences in path estimates among 
the models were identified in the between - subscale comparisons. The findings did not support the presence of differential effects of physiological symptoms of anxiety, worry/oversensitivity, and social concerns/concentration subscale ratings on youth functional impairment as measured by the CGAS and the CSR at posttreatment. As such, the total scores of youth anxiety ratings measured by the youth and parent respective versions of the RCMAS were used in all models tested in this study.

All model tests in this study were pursued using the SEM framework in Mplus 6, unless stated otherwise.

\section{Main Analyses}

\section{Levels of Anxiety Symptoms}

The first objective in this study was to test the linkages between different levels of anxiety (i.e., low, medium, and high) and youth functional impairment ratings at posttreatment and follow up. Three dummy variables were created to indicate three groups of youths who reported low, medium, and high levels of anxiety at posttreatment. The high level of anxiety was defined as one standard deviation above the sample mean on anxiety symptoms; the low level of anxiety was defined as one standard deviation below the sample mean on anxiety symptoms; and the medium level of anxiety was defined as the scores between low and high levels of anxiety. Each estimated model in these analyses was just-identified and thus no fit indices are reported.

Youth ratings. Table 3 presents unstandardized paths coefficients and their associated statistics, representing mean differences between the groups for youth completed measures (i.e., RCMAS, MASC). As a reminder, high scores on the CGAS mean 'less impaired.' As shown in Table 3, at posttreatment, using the RCMAS, youths 
who reported either low or medium on anxiety levels scored significantly higher on the CGAS $(\beta=9.67, p<.001 ;$ or $7.24, p=.001$, respectively) and lower on the CSR $(\beta=$ $2.17, p<.001$; or $-1.87, p=.002$, respectively), compared to youths who reported high anxiety levels. Similar results were found using MASC. Youths who reported low or medium anxiety levels scored significantly higher on the CGAS $(\beta=8.16, p=.002$, or $8.72, p<.001$, respectively) and lower on the CSR $(\beta=-1.56, p=.017)$, compared to youths who reported high anxiety levels. In all cases, the direction of the mean difference was toward decreased impairment vis-à-vis decreased anxiety symptom severity (see Table 3). The significant difference effects were maintained when the Holm modified Bonferroni method (Jaccard, 1998) was applied to control the experiment-wise error rate at 0.05 .

The evaluation of mean differences between low, medium, and high anxiety groups for youth completed measures (i.e., RMCAS and MASC) at posttest with respect to the CGAS and CSR scores at follow up was pursued next. No statistically significant results were revealed in these analyses.

Parent ratings. Table 4 presents unstandardized paths coefficients and their associated statistics, representing mean difference between the groups for parent completed measures (i.e., RCMAS/P). As shown in Table 4, youths whose parent rated either low or medium on anxiety levels scored significantly higher on the CGAS ( $\beta=$ 16.95 or $9.10, p<.001$, respectively) than youths whose parent rated high on anxiety levels. Additionally, youths whose parents rated low on anxiety levels scored significantly higher on the CGAS ( $\beta=7.86, p<.001)$, compared to youths whose parents rated medium on anxiety levels. The significant difference effects were maintained when 
the Holm modified Bonferroni method (Jaccard, 1998) was applied to control the experiment-wise error rate at 0.05 .

Statistically significant results were also found with the CGAS ratings at follow up, revealing that compared to youths whose parents rated high on anxiety levels, the beta coefficient for youths whose parents rated low on anxiety levels was $8.66(p=.026)$. However, the significance of this difference effect was not maintained when the Holm modified Bonferroni method (Jaccard, 1998) was applied to control the experiment-wise error rate at 0.05 .

With respect to the posttreatment CSR, youths whose parents rated low or medium on anxiety levels scored significantly lower $(\beta=-1.97, p=.001$; or $-1.52, p=$ .007 , respectively), compared to youths whose parents rated high on anxiety levels. Similar results were found at follow up, revealing that compared to youths whose parents rated medium or high on anxiety levels, youths whose parents rated low on anxiety levels scored significantly lower on $\operatorname{CSR}(\beta=-0.89, p=.001$, or $-1.12, p=.008$, respectively). The significant difference effects were maintained when the Holm modified Bonferroni method (Jaccard, 1998) was applied to control the experiment-wise error rate at 0.05 .

In all cases, using parents' ratings of youth anxiety, the direction of the mean difference was toward decreased impairment vis-à-vis decreased anxiety symptom severity (see Table 4).

\section{Incremental Validity of the CGAS}

The second objective of this study was to evaluate incremental validity of the CGAS related to the CSR at the posttreatment and 12-month follow up. Using SPSS, a two-step linear regression was pursued by entering anxiety symptom severity ratings as 
measured by the youth and parent rated RCMAS first, followed by entering the global impairment rating measured by the CGAS as a predictor of the CSR scores. In addition to RCMAS, MASC was also used as a measure of anxiety symptom severity. The criterion for an adequate incremental validity of the CGAS was a statistically significant difference between the values of adjusted $R^{2}$ for the first and second steps of the model (Cohen, 1992). ${ }^{1}$

Combined CSR. Table 5 presents relevant adjusted $\mathrm{R}^{2} \mathrm{~s}$ and their associated statistics for the combined CSR (i.e., based on youth's and parent's views of impairment). As shown in Table 5, at posttreatment, using the RCMAS, the results revealed the adjusted $R^{2}$ for the first step of the model to equal 0.17 and the addition of the posttreatment CGAS ratings with the second step resulted in an adjusted $R^{2}$ of 0.40 . The $R^{2}$ change equaled 0.23 , and this was significant $(p<.001)$. Similar results were found using the MASC (i.e., the $R^{2}$ change $=0.35, p<.001$ ). Using the RCMAS/P, the results revealed the adjusted $R^{2}$ for the first step of the model to equal 0.14 and the addition of the posttreatment CGAS ratings with the second step resulted in an adjusted $R^{2}$ of 0.38 . The $R^{2}$ change equaled 0.23 , and this was significant $(p<.001)$.

At the follow up, using the RCMAS, the adjusted $R^{2}$ for the first step of the model was equal to 0.06 and the addition of the posttreatment CGAS ratings with the second step resulted in an adjusted $R^{2}$ of 0.25 . The $R^{2}$ change equaled 0.19 , and this was significant $(p<.001)$. Similar results were found using the MASC (i.e., $R^{2}$ change $=0.21$, $p<.001)$. Using the RCMAS/P, the adjusted $R^{2}$ for the first step of the model to equal 0.09 and the addition of the CGAS ratings with the second step resulted in an adjusted $R^{2}$ of 0.25 . The $R^{2}$ change equaled 0.16 , and this was significant $(p<.001)$. 
Overall, these results suggest incremental validity of the CGAS related to the combined CSR scores at posttreatment and follow up.

ADIS-C CSR. Table 6 presents relevant adjusted $\mathrm{R}^{2} \mathrm{~s}$ and their associated statistics for ADIS-C CSR (i.e., based on youth's view of impairment). As shown in Table 6 , at posttreatment, using the RCMAS, the results revealed the adjusted $R^{2}$ for the first step of the model to equal 0.15 and the addition of the posttreatment CGAS ratings with the second step resulted in an adjusted $R^{2}$ of 0.20 . The $R^{2}$ change equaled 0.06 , and this was significant $(p=.001)$. At follow up, results revealed the adjusted $R^{2}$ for the first step of the model to equal 0.08 and the addition of the posttreatment CGAS ratings with the second step resulted in an adjusted $R^{2}$ of 0.13 . The $R^{2}$ change equaled 0.05 , and this was also significant $(p=.024)$. Similar results were found using the MASC (i.e., the $R^{2}$ change equaled $0.06, p=.001$; and $0.09, p=.003$, at the posttreatment and the follow up, respectively). These results suggest incremental validity of the CGAS related to the ADIS-C CSR scores at both posttreatment and follow up.

ADIS-P CSR. Table 7 presents relevant adjusted $\mathrm{R}^{2} \mathrm{~s}$ and their associated statistics for ADIS-P CSR (based on parent's view of impairment). As shown in Table 7, at posttreatment, using the RCMAS/P, the results revealed that the adjusted $R^{2}$ for the first step of the model to equal 0.15 and the addition of the posttreatment CGAS ratings with the second step resulted in an adjusted $R^{2}$ of 0.39 . The $R^{2}$ change equaled 0.24 , and this was significant $(p<.001)$. At follow up, results revealed that the adjusted $R^{2}$ for the first step of the model to equal 0.09 and the addition of the posttreatment CGAS ratings with the second step resulted in an adjusted $R^{2}$ of 0.17 . The $R^{2}$ change equaled 0.09 , and 
this was also significant $(p=.009)$. Overall, these findings suggest incremental validity of the CGAS related to the ADIS-P CSR scores at both posttreatment and follow up. Functional Impairment Mediation

The third objective of this dissertation was to test the proposed functional impairment mediation by anxiety symptom reductions. The conceptual model of the proposed mediation is depicted in Figures 1. A predictor variable of pretreatment anxiety symptoms (e.g., Pretreatment Anxiety in Figure 1) was assumed to impact the outcome variables of youth functional impairment as measured by the CGAS and the CSR at posttreatment (e.g., Figure 1, path $j$ and $k$ ) and at follow up (e.g., Figure 1, path $l$ and $m$ ), respectively. Paths $a$ reflects impact of the focal independent variable (e.g., Pretreatment Anxiety in Figure 1) on the mediator (e.g., Posttreatment Anxiety in Figure 1). Paths $a$ and $n$ indicates a traditional autoregressive effect representing change in anxiety symptoms from pre to posttreatment and posttreatment to follow up, respectively, as an effect of intervention collapsed across PCBT and GCBT treatment conditions. Paths $o$ and $p$ (also in Figure 1) also reflect traditional autoregressive effect representing change in functional impairment measured by the CGAS and CSR, respectively, from post to follow up as an effect of intervention collapsed across PCBT and GCBT treatment conditions.

Paths $b$ and $c$ (also in Figure 1) reflect the extent to which changes in the respective mediator (e.g., Posttreatment Anxiety) are associated with changes in the primary and secondary outcome variables at posttreatment (e.g., Impairment as measured by Posttreatment CGAS and Impairment as measured by Posttreatment CSR in Figure 1) from pre to posttreatment. Paths $h$ and $i$ reflect the extent to which changes in anxiety 
symptoms (e.g., Follow Up Anxiety) are associated with changes in measures of impairment from posttreatment to follow up (e.g., Impairment as measured by Follow Up CGAS and Impairment as measured by Follow Up CSR in Figure 1). Each path represents contemporaneous reciprocal causality (Silverman et al., 2009), in that (a) changes in youth anxiety are assumed to impact changes in youth functional impairment, and (b) changes in youth functional impairment are assumed to impact changes in youth anxiety simultaneously.

Paths $d$ and $e$ reflect lagged effects, estimating the extent to which changes in the youth anxiety from pretreatment to posttreatment (e.g., Posttreatment Anxiety in Figure 1) are associated with changes in youth functional impairment from posttreatment to follow up (e.g., Impairment as measured by Follow Up GCAS and Impairment as measured by Follow Up CSR in Figure 1, respectively). Paths $f$ and $g$ also reflect lagged effects, estimating the extent to which changes in youth functional impairment variable from pretreatment to posttreatment (e.g., Impairment as measured by Posttreatment CGAS in Figure 1) are associated with changes in youth anxiety from posttreatment to follow up (e.g., Follow Up Anxiety in Figure 1, path $f$ ).

With respect to mediation effects using the youth and parent models, the joint significance test was used to examine these effects as recommended by MacKinnon, Lockwood, Hoffman, West, and Sheets (2002). As such, the key paths of interest are $a$ through $e$. Paths $a, b$, and $c$ need to be statistically significant to conclude that posttreatment anxiety mediates, to some extent, youth functional impairment as measured by the CGAS and CSR at posttreatment, and paths $a$, $d$, and $e-$ at follow up. 
Youth ratings. Figure 2 represents the mediation model which was tested using the youth's self-ratings of anxiety (i.e., RCMAS). The model yielded a good fit to the data. The overall chi square test of model fit was not statistically significant $(\chi 2(6)=$ $5.04, p>.05)$. The CFI was 1.00 , the RMSEA was $<.001$, and the $p$ value for the test of close fit was 0.77 . More focused tests of fit revealed no theoretically meaningful or sizeable modification indices. The standardized residuals indicate the proportion of unexplained variance in the endogenous variables. The variables in the model were able to account for $24 \%$ of the variance in the RCMAS at post, $22 \%$ of the variance in the CGAS at post, $21 \%$ of the variance in the CSR at post, $37 \%$ of the variance in the CGAS at follow up, and $10 \%$ of the variance in the CSR at follow up.

With respect to youth functional impairment mediation using the CGAS as outcome, path $a$ and path $b$ were both statistically significant (path $a=0.43, p<.001$, $95 \%$ CI $[0.30,0.55]$; path $b=-0.57, p=<.001,95 \%$ CI [-0.83, -0.32$])$, suggesting that the hypothesized mediator of post RCMAS significantly mediated post CGAS. Given these findings, the total indirect effect of treatment on improvement in functional status was $-0.25(p<.001)$, and the total effect of treatment on impairment was $-0.36(p=$ $.004)^{2}{ }^{2}$ Similar results were found using the MASC (i.e., path $a=0.45, p<.001,95 \%$ CI $[0.33,0.56]$; path $b=-0.20, p<.001,95 \%$ CI $[-0.31,-0.09]$; the total indirect effect $=-$ $0.09, p=.002)$.

With respect to youth functional impairment mediation using the CSR as outcome, path $a$ and path $c$ were both statistically significant (path $c=0.16, p<.001$, $95 \% \mathrm{CI}[0.10,0.23])$, suggesting that the hypothesized mediator of post RCMAS significantly mediated post CSR. Given these findings, the total indirect effect of 
treatment on functional impairment was $0.07(p<.001)$, and the total effect of treatment on impairment as measured by the CSR at post was not statistically significant. Similar results were found using the MASC (i.e., path $c=0.03, p=.015,95 \%$ CI $[0.006,0.06]$; the total indirect effect $=0.13, p=.01$ ). That is, both PCBST and GCBT resulted in youth having significant improvements in anxiety symptoms, which in turn mediated significant improvements in youth functional status as measured by both the CGAS and CSR at posttreatment, as reported by youths.

With respect to youth functional impairment mediation using youth self-rating of anxiety and the CGAS and CSR at follow up as outcome, only path $a$ was statistically significant. As such, there is no evidence that either RCMAS or MASC at post mediates youth functional impairment as measured by the CGAS and CSR at the follow up.

Parent ratings. Figure 3 represents the model which was tested using the parent's ratings of youth anxiety (i.e., RCMAS/P). The model yielded a good fit to the data. The overall chi square test of model fit was not statistically significant $(\chi 2(6)=6.41, p>.05)$. The CFI was 1.00 , the RMSEA was 0.019 , and the $p$ value for the test of close fit was 0.64. More focused tests of fit revealed no theoretically meaningful or sizeable modification indices. The standardized residuals indicate the proportion of unexplained variance in the endogenous variables. The variables in the model were able to account for $32 \%$ of the variance in RCMAS/P at post, $34 \%$ of the variance in the CGAS at post, $19 \%$ of the variance in the CSR at post, $54 \%$ of the variance in the CGAS at follow up, and $11 \%$ of the variance in the CSR at follow up.

With respect to youth functional impairment mediation using the CGAS as outcome, it was found that path $a$ and path $b$ were both statistically significant (path $a=$ 
$0.54, p<.001,95 \%$ CI [0.41, 0.67]; path $b=-1.16, p<.001,95 \%$ CI [-1.45, -0.86$])$, suggesting that the hypothesized mediator of post RCMAS/P significantly mediated post CGAS. Given these findings, the total indirect effect of treatment on functional status was $-0.62(p<.001)$; the total effect of treatment on impairment was $-0.44(p=.012)$.

With respect to youth functional impairment mediation using the CSR as outcome, it was found that both path $a$ and path $c$ were statistically significant (path $c=$ $0.19, p<.001,95 \% \mathrm{CI}[0.12,0.26])$, suggesting that the hypothesized mediator of post RCMAS significantly mediated post CSR. Given these findings, the total indirect effect of treatment on functional impairment was $0.10(p<.001)$, and the total effect of treatment on impairment as measured by the CSR at was not statistically significant.

With respect to youth functional impairment mediation using parent measures of youth anxiety and the CGAS and CSR at follow up as outcome, only path $a$ was statistically significant. As such, there is no evidence that the RCMAS/P at posttest mediates youth functional impairment as measured by the CGAS and CSR at follow up.

\section{Directionality of Change}

The next objective of this study was to evaluate the directionality of change in the relation between anxiety symptom reduction and youth functional impairment (i.e., symptom-to-impairment, impairment-to-symptom, or bidirectional). Figures 4 and 5 represent the youth and parent models, respectively, which were tested. The key paths of interest are $b$ through $g$.

As noted earlier, paths $b$ and $c$ represent contemporaneous change and estimate the extent to which (a) changes in youth anxiety are assumed to impact changes in youth functional impairment; and (b) changes in youth functional impairment are assumed to 
impact changes in youth anxiety during the same time period (i.e., from pretreatment to posttreatment). The evaluation these reciprocal paths could not be pursued simultaneously due to the issue of underidentification (Bollen, 1989; Kenny, 1979). As such, it was necessary to run the youth and parent models twice: once where paths $b$ and $c$ flowed from youth anxiety to the youth functional impairment variables; and a second time where the paths flowed from youth functional impairment to youth anxiety.

The causal flow of paths $b$ and $c$ between the variables of anxiety and impairment in youth and parent models was demonstrated by the previously reported findings. When the causal direction was reversed, the paths $b$ and $c$ were also statistically significant, whether using the RCMAS, (path $b=-0.14, p<.001,95 \%$ CI [-0.20, -0.08]; path $c=$ $0.86, p<.001,95 \%$ CI $[0.46,1.26])$, the MASC (path $b=-0.33, p=.001,95 \%$ CI $[-0.54$, -0.13]; path $c=1.34, p<.001,95 \%$ CI $[0.22,2.46]$ ), or the RCMAS/P (path $c=-0.18, p$ $<.001,95 \%$ CI $[-0.24,-0.13]$; path $c=0.70, p<.001,95 \%$ CI $[0.42,0.98])$. Which direction the causality should flow however is ambiguous because in both cases the same research question is being tested: Do changes in youth anxiety produce changes in youth functional impairment contemporaneously? As such, the significant causal flow of these paths one way and the other does not provide an answer with respect to the directionality of change between variables of youth anxiety and impairment in these models. According to Jaccard \& Jacoby (2010), in the analyses of bidirectional causal relationships, the evaluation of lagged effects between the variables of interest is important given that time interval is necessary to explain how much one variable influences the other, and vice versa. 
As discussed earlier, paths $d$ through $g$ (in Figure 4) reflect lagged effects, estimating the extent to which (a) changes in the youth anxiety from pretreatment to posttreatment (e.g., Post RCMAS in Figure 4) are associated with changes in youth functional impairment measured from posttreatment to follow up (e.g., Follow Up GCAS in Figure 4, path $d$ ); and (b) the extent to which changes in youth functional impairment variable from pretreatment to posttreatmet (e.g., Post CGAS in Figure 4) are associated with changes in youth anxiety from posttreatment to follow up (e.g., Follow Up RCMAS in Figure 4, path $f$ ). As such, paths $d$ through $g$ need to be statistically significant to declare the bidirectionality in the symptom-to-impairment causal relation.

The results revealed that across all model tests and informants, paths $d$ though $g$ were not statistically significant (see Figures 4 and 5). Similar to Silverman et al. (2009), it was assumed that the conclusions vis-à-vis the lagged effects are the same, regardless of the way the model is represented in terms of paths $b$ and $c$. Thus, the study did not provide empirical evidence for a reverse causal mechanism in the relation between anxiety symptom reduction and change in youth functional impairment measured by either the CGAS or the CSR.

Given that the paths $d$ through $g$ in both youth and parent models were not statistically significant, the measures of both youth anxiety and youth functional impairment at the follow up were not included in all following model tests.

\section{Moderator Analyses}

The fourth objective of this study, to evaluate the respective moderating effects of youth's age, sex, and ethnicity on the mediated relation between youth anxiety symptom 
reduction and functional impairment, was pursued using a product term approach ${ }^{3}$ and a series of multiple group analyses. ${ }^{4}$

Youth age. Given that youth age is a continuous variable, its moderating effect on the youth functional impairment mediation by treatment gains was evaluated using the product term approach (Jaccard \& Wan, 1996; Jaccard, Turrisi \& Wan, 1990). Figure 6 and 7 represents the youth and parent models, respectively, which were tested. Of specific interest are paths $d$ and $e$, representing differential effects of youth age (e.g., Post RCMAS*Age in Figure 6) on the slope for the regression of the primary and secondary outcome variables (e.g., Post CGAS at and Post CSR in Figure 6) onto the mediator (e.g., Post RCMAS in Figure 6). As such, paths $d$ and $e$ need to be statistically significant to declare an interaction effect of youth age on the mediated relation between anxiety symptom reduction and youth functional impairment at posttreatment.

Figure 6 represents the model which was tested with youth completed measures of anxiety (i.e., RCMAS). The model yielded a good fit to the data. The overall chi square test of model fit was not statistically significant $(\chi 2(3)=2.62, p>.05)$. The CFI was 1.00 , the RMSEA was $<.001$, and the $p$ value for the test of close fit was 0.63 . More focused tests of fit revealed no theoretically meaningful or sizeable modification indices. Paths $d$ and $e$ were not statistically significant $(p>.05)$. Similar results were found using the MASC, suggesting a lack of significant interaction effects of youth age on the mediated relation between anxiety symptom reduction and youth functional impairment at posttreatment, using youth self-ratings of anxiety (i.e., RCMAS, MASC).

Figure 7 represents the model which was tested with parent ratings of youth anxiety (i.e., RCMAS/P). This model also yielded a good fit to the data. The overall chi 
square test of model fit was not statistically significant $(\chi 2(3)=5.41, p>.05)$. The CFI was 0.99 , the RMSEA was 0.066 , and the $p$ value for the test of close fit was 0.30 . More focused tests of fit revealed no theoretically meaningful or sizeable modification indices. Paths $d$ and $e$ were not statistically significant $(p>.05)$, suggesting a lack of significant interaction effect of youth age on the mediated relation between anxiety symptom reduction and youth functional impairment at posttreatment using parent rating of youth anxiety (i.e., RCMAS/P).

In addition to the product term analyses, a series of multiple groups was pursued based on middle childhood, late childhood, and adolescence. No evidence for moderation was found using either youth or parent ratings of youth anxiety (i.e., RCMAS, MASC, or RCMAS/P).

Youth sex. To assess a moderating effect of youth sex on the youth functional impairment mediation by treatment gains, a series of multiple group analyses was pursued. Two dummy variables were constructed to indicate boys $(n=98)$ and girls $(n=$ 85 ) in the study's sample. Using youth ratings of anxiety (i.e., RCMAS and MASC), a multiple group model with regression paths constrained to be equal across youth sex provided a good fit. The chi square test of model fit was not statistically significant $(\chi 2$ $(21)=22.41, p>.05)$. The CFI was .99 , the RMSEA was 0.03 , and the $p$ value for the test of close fit was 0.64 . Relaxing the path equality constraint did not significantly improved the model fit, $\chi 2$ difference $(13, N=183)=12.59, p>.05$. Similar results were found using the MASC (i.e., $\chi 2$ difference $[13, N=183]=11.47, p>.05$ ).

Using parent ratings of youth anxiety (i.e., RCMAS/P), a multiple group model with regression paths constrained to be equal across age groups provided an acceptable 
fit. The chi square test of model fit was not statistically significant $(\chi 2(21)=30.60, p>$ .05 ), the CFI was .96 , the RMSEA was 0.07 , and the $p$ value for the test of close fit was 0.25. Relaxing the path equality constraint did not lead to a significantly better model fit, $\chi 2$ difference $(13, N=183)=17.02, p>.05$. As such, using multiple group analyses approach, the results of both the youth and parent models suggest a lack of significant interaction effects of youth sex on the mediated relation between anxiety symptom reduction and youth functional impairment at posttreatment across all informants.

Youth ethnicity. A moderating effect of youth ethnicity on the youth functional impairment mediation by treatment gains was tested using multiple group analysis method. Given the high proportion of Hispanic families in the sample (75\%), only two dummy variables were constructed to indicate youth ethnicity: Hispanics $(n=139)$ and non-Hispanics $(n=44)$. Using youth ratings of anxiety (i.e., RCMAS and MASC), a multiple group model with regression paths constrained to be equal across youth ethnicity groups provided an acceptable fit. The chi square test of model fit was not statistically significant $\left(\chi^{2}(21)=32.05, p>.05\right)$. The CFI was .94 , the RMSEA was 0.08 , and the $p$ value for the test of close fit was 0.20 . Relaxing the path equality constraint did not lead to a significantly better model fit, $\chi^{2}$ difference $(13, N=183)=17.82, p>.05$. Similar results were found using the MASC (i.e., $\chi^{2}$ difference [13, $\left.N=183\right]=15.72, p>.05$.

Using parent ratings of youth anxiety (i.e., RCMAS/P), a multiple group model with regression paths constrained to be equal across age groups provided a good fit. The chi square test of model fit was not statistically significant $\left(\chi^{2}(21)=22.10, p>.05\right)$, the CFI was 1.00 , the RMSEA was 0.02 , and the $p$ value for the test of close fit was 0.65 . Relaxing the path equality constraint did not lead to a significantly better model fit, $\chi^{2}$ 
difference $(13, N=183)=13.44, p>.05$. As such, using multiple group analyses approach, the results of both the youth and parent models suggest a lack of significant interaction effects of youth ethnicity on the mediated relation between anxiety symptom reduction and youth functional impairment at posttreatment across all informants.

\section{Informant Agreement}

The sixth and final objective of this study was to evaluate an agreement (or lack thereof) between youths and parents' views of youth functional impairment measured by ADIS/C CSR and ADIS/P CSR, respectively, vis-à-vis youth anxiety reduction as measured by the youth (i.e., RCMAS, MASC) and their parents (i.e., RCMAS/P). Figures 8 and 9 represent the youth and parent models, respectively, which were tested. The joint significance test as recommended by MacKinnon, Lockwood, Hoffman, West, and Sheets (2002) was used to examine mediation effects in the youth and parent models. As such, the key paths of interest are paths $a$ and $b$. That is, paths $a$ and $b$ need to be statistically significant to conclude that the RCMAS at posttreatment mediates, to some extent, youth functional impairment as measured by either the post ADIS/C CSR (in Figure 8) or the post ADIS/P CSR (in Figure 9).

Two separate models with the CSR based on youth and parent views of impairment at posttreatment (e.g., Post ADIS/C CSR in Figure 8 and Post ADIS-P CSR in Figure 9, respectively) as outcome were tested separately. Both models were justidentified and as such no fit indices are reported.

Youth ratings. Using youth's self-ratings of anxiety (i.e., RCMAS in Figure 8) and impairment (i.e., ADIS/C CSR also in Figure 8), the model test revealed that the 
variables in the model were able to account for $24 \%$ of the variance in the RCMAS at post and $22 \%$ of the variance in the ADIS/C CSR at post.

With respect to youth functional impairment mediation using the ADIS/P CSR as outcome, path $a$ and $b$ were both statistically significant (path $a=0.44, p<.001,95 \% \mathrm{CI}$ $[0.32,0.56]$; path $b=0.12, p=.001,95 \%$ CI $[0.05,0.20])$, suggesting that the hypothesized mediator of the RCMAS at post significantly mediated the ADIS/C CSR at post. Given these findings, the total indirect effect of treatment on functional impairment was $0.06(p=.002)$, and the total effect of treatment on impairment as measured by the ADIS/C CSR at posttreatment was not statistically significant. Similar results were found using the MASC (i.e., path $a=0.44, p<.001,95 \%$ CI $[0.33,0.55]$; path $b=0.03, p=$ $.004,95 \% \mathrm{CI}[0.01,0.05]$; the total indirect effect $=0.01, p=.005)$.

Parent ratings. Using parent ratings of youth anxiety (i.e., the RCMAS/P in Figure 9) and impairment (i.e., ADIS/P CSR also in Figure 9), the model test revealed that the variables in the model were able to account for $31 \%$ of the variance in the RCMAS at post and $24 \%$ of the variance in the ADIS/P CSR at post.

With respect to youth functional impairment mediation using the ADIS/P CSR as outcome, path $a$ and $b$ were both statistically significant (path $a=0.56, p<.001,95 \% \mathrm{CI}$ $[0.44,0.68]$; path $b=0.21, p<.001,95 \%$ CI $[0.13,0.28])$, suggesting that the hypothesized mediator of RCMAS at post significantly mediated the ADIS-P CSR at post. Given these findings, the total indirect effect of treatment on functional impairment was $0.12(p<.001)$, and the total effect of treatment on impairment as measured by the ADIS/P CSR at post was not statistically significant. 
To evaluate whether youth and parents agree in their views of change in youth functional impairment vis-à-vis anxiety symptom reduction, path estimates were examined, and informal comparisons of path estimates of the models were made. No theoretically meaningful differences in path estimates between the youth and parent models were identified in the between-source comparisons, suggesting a relative informant agreement with respect to change in youth functional status vis-à-vis anxiety reduction at posttreatment. 


\section{CHAPTER V.}

\section{DISCUSSION}

The present study aimed to evaluate: (1) the relation between low, medium, and high levels of anxiety symptom severity and youth functional impairment ratings as measured by both the C-GAS (Shaffer et al., 1983) and the CSR (Silverman \& Albano, 1996); (2) whether functional impairment ratings measured by the CGAS predict impairment above and beyond measures of anxiety severity symptoms, indicating incremental validity of the CGAS; (3) whether the impact of psychosocial treatments aimed at reducing youth phobic and anxiety disorders in a PCBT (targeting parenting behaviors and the parent-youth relationship) and a GCBT (targeting youth social skills behaviors and the peer-youth relationship) approach mediate change significantly in youth functional status; (4) whether the proposed mediated relation between treatment outcome (i.e., anxiety symptom reduction) and youth functional impairment vary as a function of youth age, sex, and ethnicity; (5) the directionality of change between anxiety symptom reduction and youth functional impairment; and (6) an agreement (or lack thereof) between youths and their parents in their views of youth impairment vis-à-vis anxiety symptom reduction.

\section{Summary of Dissertation Findings}

With respect to the linkages between different levels of anxiety and functional impairment, the findings revealed that at posttreatment, youths who scored either low or medium on anxiety reported significant reductions in functional impairment, compared to youth who scored high on anxiety. The pattern of findings was consistent across all

posttreatment measures of youth anxiety (i.e., pre and post RCMAS, MASC, and 
RCAMS/P) and impairment outcome (i.e., post CGAS and CSR). In contrast to expectation, these effects were maintained at follow up only when using parent ratings of youth anxiety (i.e., pre and post RCMAS/P) and the combined CSR as outcome (i.e., based on both youth's and parent' views of impairment).

The findings also revealed that CGAS ratings predicted impairment in youth functioning above and beyond measures of anxiety severity symptoms, demonstrating incremental validity of the CGAS. As expected, the effects were consistent across all assessment points (i.e., posttreatment and follow up), measures of anxiety (i.e., RCMAS, MASC, RCMAS/P), and impairment outcome (i.e., CGAS, combined CSR, ADIS-C CSR [i.e., based on youth's view of impairment], and ADIS-P CSR [i.e., based on parent's view of impairment]).

With respect to youth functional mediation by treatment gains, results revealed that psychosocial treatments in both PCBT and GCBT produced significant improvements in anxiety symptoms, which in turn reduced significantly youth functional impairment at posttest, across all measures of youth anxiety and impairment. In contrast to expectations, these effects were not maintained at follow up.

With respect to the directionality of change between anxiety symptom reduction and improvement in youth functional impairment, no evidence for a reverse causal path was revealed. In contrast to expectations, the lagged effects that linked changes in anxiety symptoms and functional impairment over time were not significant. These findings suggest that the direction of change between the variables is symptom-to-impairment rather than vice versa. 
No evidence was observed for the respective moderating effects of youths' age, sex, or ethnicity analyses on the relation between anxiety symptom reductions and improvement of youth functioning. Results indicated that the strength or direction of the posttreatment youth functional status mediation by treatment gains did not vary as a function of these specific youth background characteristics.

With respect to informant agreement, no evidence for discrepancies in youths' and parents' views of change in youth functional impairment vis-à-vis anxiety reduction was observed. In contrast to expectations, informal comparisons of youth and parent model path estimates, coupled with the uniform results of mediation and moderation model tests across both informants, did not reveal theoretically meaningful differences. As such, the results indicated a relative agreement between youths and parents in their perception of youth functional status mediated by treatment gains.

\section{Contribution of the Present Study and Implications}

The present study is important on theoretical, empirical, and clinical levels. Its main contributions and potential implications on each of these levels are elaborated upon in more detail in the following sections.

Theoretical implications. By evaluating the relation between different levels of youth anxiety and youth impairment, the current study extends the theoretical literature regarding the issue of arbitrary metrics inherent in testing of clinical significance of psychosocial treatment outcome (Jaccard \& Blanton, 2009; Kazdin, 1999). Findings at what level of severity (e.g., low, medium, or high) youth anxiety test scores are linked to meaningful real-life events - namely, observable and important change in youths' functional status across major domains of functioning - help to reduce metric 
arbitrariness. This is because such findings convey a theoretical understanding of how change in anxiety translates into the change in the youth's real world experiences. That is, the findings not only demonstrate statistically significant mean changes on a measure of interest (e.g., anxiety), but also show that those changes have meaningful consequences for youth in terms of their ability to effectively care for one's self, interact with others, and meet demands of social role performance and role satisfaction in various settings and situations. Although not sufficient to generate nonarbitrary metrics, the study's findings have a potential to ascertain clinical significance of treatment outcome. As noted by Kazdin (1999), evaluation of whether change in symptoms (such as anxiety) has practical and real world implications should be one of the criteria "along which clinical significance ought to be evaluated" (p. 334).

Findings with respect to both the CGAS' utility to predict youth functional impairment independently from anxiety symptom severity measures and the lack of a reverse causal mechanism between variables of symptoms and impairment add to theoretical literature concerning the conceptualization of functional impairment. They suggest that although symptoms (such as anxiety) are closely linked with and have often been used as a proxy for impairment, these two concepts are not interchangeable and thus should be evaluated independently (Bird, 1999; Kazdin, 1999). Empirical evidence for incremental validity of the CGAS also advances theoretical knowledge. It suggests that for treatment gain ascertainment, it is insufficient to detect youths who no longer meet symptomatic criteria for one or more psychological disorders. Instead, it is just as important to evaluate improvement in impairment associated with, but assessed independently from, symptom reductions (Bird et al., 1993). 
Moreover, the current study extends past outcome research theoretically by evaluating the mediated relation between variables measuring youth anxiety and functional impairment. Such evaluations allow for verification of fit between theoretical research and clinical application of treatment (Kazdin, 2001). Furthermore, revealing how change in impairment is produced can help to advance theoretical knowledge about the nature of change produced in psychosocial interventions and the specific variable (i.e., the mediator) that trigger this critical change. Once this specific variable is identified, it can be included as the key component in the therapeutic processes to enhance the design and delivery of existing treatment strategies. As such, elucidating the mediator of positive change in youth functional status can potentially inform theory construction in youth anxiety treatment to render maximally effective interventions. That is, empirical knowledge about what leads to meaningful changes in youth functional impairment, and what does not can help move the field toward research of innovative treatment models that will not only reduce youth's symptoms but also evoke practical and palpable improvements in lives of youths and their families (Kazdin, 1999).

It is interesting that significant mediation of functional impairment by treatment gains were not maintained at follow up. One explanation for this finding is that both anxiety and impairment in functioning are unstable psychological constructs that can be influenced by a number of individual (e.g., competence, personal characteristics, and communication styles) and contextual (e.g., the culture of the environment and society's goals and expectations) factors interacting in multiple and complex ways (APA, 2000). Thus, despite the change in the respective means of anxiety symptoms from pre to posttreatment and functional impairment ratings from posttreatment to follow up (see 
Table 2), the strength of the relation between anxiety symptoms reduction and change in functional impairment is likely to decrease over time (Jeremy Pettit, personal communication, September 30, 2011). Another possibility is that given a 12-month interval between assessment points, the mediating relation between symptom reduction and change in functional impairment may become contaminated by moderating variables that the present study did not account for. Future studies replicating the present results should address this and other related issues.

Empirical implications. The present study's findings have important contributions and potential implications on the empirical level. They add to past research by demonstrating the utility of the CGAS in discriminating between clinical and nonclinical groups. They also extend past research by revealing incremental validity of the CGAS, which suggests that the CGAS predicts youth functional impairment, above and beyond measures of anxiety symptoms.

The present study contributes to past research empirically by providing further support for the efficacy of psychosocial treatments to reduce the severity of youth anxiety and associated with it functional impairment (Silverman et al., 2008). Given the field's relative inattention to change in youth functional status vis-à-vis successful treatment outcomes, the present study's findings also extend past research. They suggest that irrespective of youth age, sex, and ethnical background, reductions in the severity of anxiety symptoms mediate significantly therapeutic effects on youth functional status, but only at posttest. Future outcome research is warranted to continue filling empirical knowledge gap with regard to the relation of change in symptom and impairment in youth's daily functioning. 
Given past research findings that parents and their youth often disagree on the presence of diagnostic conditions, irrespective of diagnostic type (e.g., Jensen et al., 1999), the findings with regard to informant agreement advances empirical literature. As stated above, no evidence for youth and parent disagreement in their views of change in impairment vis-à-vis symptom reductions was observed. This may be due in part to how impairment in youth functioning is associated with anxiety disorders manifestations. Youth anxiety disorders can lead to persistent, pervasive, and significant personal distress and impairment in youths' school performance, friendships, and family relations (APA, 2000). Thus, it is unlikely that youths and parents would fail to both recognize and report positive change in youth functional impairment vis-à-vis anxiety symptom reduction. Further research is warranted to reconcile past and current research findings' inconsistencies.

Finally, the current study is, in part, a response to calls made in the treatment research literature regarding the need for research on directionality of change in causal relations among variables (Silverman et al., 2009). By evaluating empirically (and finding no evidence of) whether time is necessary for the changes in anxiety to work their way through and produce changes in functional impairment, the current study begins filling the knowledge gap about the complex nature of the mediated relation between the variables of anxiety symptoms and functional impairment. Future research employing more advanced research designs and methods that would test bidirectional influence dynamics among variables of interest is necessary.

Clinical implications. The study's findings have important clinical implications. They provide further evidence for the utility of cognitive behavioral treatment approaches 
(i.e., gradual exposures to anxiety provoking situations in conjunction with the use of cognitive strategies) implemented in both PCBT and GCBT to reduce significantly the severity of anxiety symptoms and associated with it functional impairment. Finding these treatment approaches are efficacious whether they are delivered to young children or adolescents, or youth with Hispanic/Latino or non- Hispanic/Latino cultural background further suggests the developmental and cultural sensitivity of the psychosocial interventions used in this study. This knowledge is important because it allows clinicians flexibility in their decision making on what CBT treatment approach/technique to implement to meet cost effectively the needs of each individual child presenting with anxiety-related issues.

Given the findings with regard to the directionality of change, this study suggests the likely utility of focusing in treatment first on symptom reduction. As the severity of anxiety improves, impairment in functioning will show improvement as well. For example, youths with social anxiety disorders are often so preoccupied with excessive worries and bothersome thoughts that they are unable to engage in many "everyday" activities involving the family, the peers, or the school. The youths' inability to engage in such activities may lead to additional problems in functioning, such as family conflicts, impaired peer relationships, and school absenteeism (Silverman \& Kurtines, 1996). As suggested by the present findings, to help youths with social anxiety disorders in treatment, it is useful to focus first on youths' symptoms by implementing evidencebased approaches to target maladaptive behavioral, cognitive and affective processes. Once symptoms are alleviated, improvement in functioning will follow because the 
youths will no longer avoid going on the family outings, socializing with friends, or attending school.

Significant findings with respect to the relation between low and medium levels of anxiety and functional impairment at posttreatment are also important on the clinical level. They suggest that youths whose anxiety problems are at low or medium level are a cause for concern, and those youths whose anxiety problem are at high level should be considered in need of mental health services. This knowledge is important because it can help clinicians to (1) better understand the phenomenology of anxiety disorders in youth, (2) prioritize interventions, (3) refine diagnostic criteria, and (4) guide their decisions with respect to resource allocation for youths and their families seeking psychological services.

\section{Limitations and Future Research Directions}

The present study has several limitations that merit considerations when interpreting the results. One limitation is that the mediator variable of anxiety symptom reduction was only measured at two time points (i.e., pretreatment to posttreatment). This is an issue because it disallowed an evaluation of whether changes in the mediator (i.e., anxiety symptom reduction at posttreatment) precede change in the outcome (i.e., youth functional impairment). Future research should be pursued that takes into account this important temporal issue by involving more frequent assessments of youth anxiety symptoms and functional impairment on a session by session basis (Kraemer, Wilson, Fairburn, \& Agras, 2002).

A second limitation is that the parent participants were mothers. Youth anxiety symptom reduction and functional impairment was thus evaluated only from participant 
mother's point of view. This is an issue because information from both parents in necessary to obtain a more complete picture of how change in youth functional impairment vis-à-vis anxiety symptom reduction manifests. Future research based on both mothers and fathers' ratings in the assessment of their youth's functional impairment is warranted.

A third limitation is that intent-to-treat analyses were not pursued in this study. This is an issue because without intent-to-treat analyses, it was not feasible to evaluate differences between treatment completers and non-completers with respect to the variables of interest. To fully understand the relation between symptom reduction and change in functional impairment among youth with disorders, future research that pursues analyses based on treatment completers and intent-to-treat is necessary.

Another limitation was the method of measurement of youth functional impairment used in this study. The use of global measures of impairment such as the CGAS and the CSR can only provide an overall assessment of problems in youth functioning, suggesting the scales' practical limitations (Fabiano et al., 2009). To better understand the nature of the association of change between youth anxiety symptoms and functional impairment, the use of specific information on impaired areas of youth functioning is perhaps necessary. Future studies using instruments to assess youth's impairment in specific domains of functioning is warranted.

Despite these limitations, the present results are encouraging. Demonstrating empirically that anxiety symptom reduction is a significant mediator of improvement in youth functioning, the study supports past research on the efficacy of CBT in both a PCBT and GCBT approach to improve not only the symptoms but also youth's everyday 
functioning at home, at school, and society at large. Evaluating empirically the directionality of change between symptoms and impairment, the current study sets the stage for future research that will provide evidence-based explanations of the impact of treatment gains on functional impairment among youth with anxiety disorders. 


\section{LIST OF REFERENCES}

Achenbach, T. M. (1991). Manual for the Child Behavior Checklist and Revised Child Behavior Profile (Revised). Department of Psychiatry, University of Vermont, Burlington.

American Psychiatric Association. (2000). Diagnostic and statistical manual of mental disorders (Revised $4^{\text {th }}$ ed.). Washington, DC: Author.

American Psychological Association. (2002). Code of ethics. Washington, DC: Author.

Angold, A., \& Costello, E. J. (1996). Toward establishing an empirical basis for the diagnosis of oppositional disorder (ODD). Journal of the American Academy of Child and Adolescent Psychiatry, 35, 1205-1212.

Angold, A., Costello, E. J., Farmer, E., Burns, B. J., \& Erkanli, A. (1999). Impaired but undiagnosed. Journal of the American Academy of Child and Adolescent Psychiatry, 38, 129-137.

Angold, A., Erkanli, A., Farmer, E. M. Z., Fairbank, J. A., Burns, B. J., Keeler, G., \& Costello, E. J. (2002). Psychiatric disorder, impairment, and service use in rural African American and White youth. Archives of General Psychiatry, 59, 893-901.

Baldwin, J. S., \& Dadds, M. R. (2007). Reliability and Validity of Parent and Child Versions of the Multidimensional Anxiety Scale for Children in Community Samples. Journal of American Academy for Child and Adolescent Psychiatry, 46, 252-260.

Baron, R. M., \& Kenny, D. A. (1986). The moderator-mediator variable distinction in social psychological research: Conceptual, strategic, and statistical considerations. Journal of Personality and Social Psychology, 51, 1173-1182.

Barrett, P. M. (1998). Evaluation of cognitive-behavioral group treatments for childhood anxiety disorders. Journal of Clinical Child Psychology, 27, 459-468.

Barrett, P. M., Dadds, M. R., \& Rapee, R. M. (1996). Family treatment of childhood anxiety: A controlled trial. Journal of Consulting and Clinical Psychology, 64, 333-342.

Beidel, D. C., Turner, S. M., \& Morris, T. L. (2000). Behavioral treatment of childhood social phobia. Journal of Consulting and Clinical Psychology, 68, 1072-1080.

Bentler, P. M. (1990). Comparative fit indexes in structural models. Psychological Bulletin, 107, 238-246. doi:10.1037/0033-2909.107.2.238. 
Berman, S. L., Weems, C. F., Silverman, W. K., \& Kurtines, W. M. (2000). Predictors of outcome in exposure-based cognitive and behavioural treatments for phobic and anxiety disorders in children. Behavior Therapy, 31, 713-731.

Bird, H. (1999). The assessment of functional impairment. In D. Shaffer, C. P. Lucas, \& J. E. Richters (Eds.), Diagnostic assessment in child and adolescent psychopathology (pp. 398-229). New York, NY, US: Guilford Press.

Bird, H. R., Canino, G., Rubio-Stipec, M., \& Ribera, J. C. (1987). Further measures of the psychometric properties of the Children's Global Assessment Scale (CGAS). Archives of General Psychiatry, 44, 821-824.

Bird, H., Davies, M., Fisher, P., Narrow, W., Jensen, P., Hoven, C., . . Dulcan, M. K. (2000). How specific is specific impairment? Journal of the American Academy of Child and Adolescent Psychiatry, 39, 1182-1189.

Bird, H. R., Shaffer, D., Fisher, P., Gould, M. S., Staghezza, B., Chen, J. Y., \& Hoven, C. (1993). The Columbia Impairment Scale (CIS): Pilot findings on a measure of global impairment for children and adolescents. International Journal of Methods in Psychiatric Research, 3, 167-176.

Blair, R. C., Higgins, J. J., Topping, M. E., \& Mortimer, A. L. (1983). An investigation of the robustness of the $t$ test to unit of analysis violations. Educational and Psychological Measurement, 43, 69-80.

Blanton, H., \& Jaccard, J. (2006). Arbitrary metrics in psychology. American Psychologist, 61, 27-41.

Bollen, K. A. (1989). Structural equations with latent variables. New York: John Wiley.

Brown, M. W., \& Cudeck, R. (1993). Alternative ways of assessing model fit. In K. Bollen \& J. Long (Eds.), Testing structural equation models (pp. 136-162). Newbury Park, CA: Sage.

Byrne, B.A. (2001). Structural equation modeling with AMOS: Basic concepts, applications, and programming. Mahwah, NJ: Lawrence Erlbaum Associates.

Cassidy, J., \& Asher, S. (1992). Loneliness and peer relations in young children. Child Development, 63, 350-365.

Chambless, D. L., Sanderson, W. C., Shoham, V., Johnson, S. B., Pope, K. S., CritsChristoph, P., . . McCurry, S. (1996). An update on empirically validated therapies. The Clinical Psychologist, 49, 5-18. 
Chambless, D. L., \& Hollon, S. D. (1998). Defining empirically supported therapies. Journal of Consulting and Clinical Psychology, 66, 7-18.

Cohen, J. (1992). A power primer. Psychological Bulletin, 112, 155-159.

Cohen, J., \& Cohen, P. (1983). Applied multiple regression/correlation analysis for the behavioral sciences (2nd ed.). Hillsdale, NJ: Erlbaum.

Cole, D. A., Peeke, L. G., Martin, J. M., Truglio, R., \& Serocynski, A. D., (1998). A longitudinal look at the relation between depression and anxiety in children and adolescents. Journal of Consulting and Clinical Psychology, 66, 451-460.

Costello, J. E., Egger, H. L., Copeland, W., Erkanli, A., \& Angold, A. (2009). The developmental epidemiology of anxiety disorders: Phenomenology, prevalence, and comorbidity. In W. K. Silverman, \& A. Field (Eds.), Anxiety Disorders in Children and Adolescents: Research, Assessment and Intervention. Cambridge University Press.

Costello, E. J., \& Shugart, M. A. (1992). Above and below the threshold: Severity of psychiatric symptoms and functional impairment in a pediatric sample.

Pediatrics, 90, 359-368.

Costello, E. J., Mustillo, S., Erkanli, A., Keeler, G., \& Angold, A. (2003). Prevalence and development of psychiatric disorders in childhood and adolescence. Archives of general psychiatry, 60, 837-844.

Dadds, M. R., James, R. C., Barrett, P. M., \& Verhulst, F. C. (2004). Diagnostic Issues. In T. H. Ollendick \& J. S. March (Eds.), Phobic and anxiety disorders in children and adolescents: A clinician's guide to effective psychosocial and pharmacological interventions (pp. 3-33). New York: Oxford University Press.

De Los Reyes, A., Alfano, C.A., \& Beidel, D.C. (2011). Are clinicians' assessments of improvements in children's functioning "global"? Journal of Clinical Child and Adolescent Psychology, 40, 281-294.

Duhig, A. M., Renk, K., Epstein, M. K., \& Phares, V. (2000). Interparental agreement on internalizing, externalizing and total behavior problems: A meta-analysis. Clinical Psychology: Science and Practice, 7, 435-453.

Endicott, J., Spitzer, R.L., Fleiss, J.L., \& Cohen, J. (1976). The Global Assessment Scale: A procedure for measuring overall severity of psychiatric disturbance. Archives of General Psychiatry, 33, 766-771. 
Essau, C. A., Conradt, J., \& Petermann, F. (2000). Frequency, comorbidity, and psychosocial impairment of anxiety disorders in German adolescents. Journal of Anxiety Disorders, 14, 263-279.

Ezpeleta, L., Reich, W., \& Granero, R. (2009). Assessment of distress associated to psychopathology in children and adolescents. Escritos de Psicología, 2, 19-27.

Ezpeleta, L., Keeler, G., Erkanli, A., Costello, E. J., \& Angold, A. (2001). Epidemiology of psychiatric disability in childhood and adolescence. Journal of Child Psychology and Psychiatry, 42, 901-914.

Fabiano, G. A., Pelham Jr., W. E., Washchbusch, D. A., Gnady, E. M., Lahey, B. B., Chronis, A. M., . . . Burrow-MacLean, L. (2006). A practical measure of impairment: Psychometric properties of the impairment rating scale in samples of children with Attention Deficit Hyperactivity Disorders and two school-based samples. Journal of Clinical Child \& Adolescent Psychology, 35, 369-385.

Flannery-Schroeder, E. C., \& Kendall, P. C. (2000). Group versus individual cognitive behavioral treatment for youth with anxiety disorders: A randomized clinical trial. Cognitive Therapy and Research, 24, 251-278.

Ginsburg, G. S., \& Silverman, W. K. (2000). Gender role orientation and fearfulness in children with anxiety disorders. Journal of Anxiety Disorders, 14, 57-68.

Haynes, S. N., \& Lench, H. C. (2003). Incremental validity of new clinical assessment measures. Psychological Assessment, 15, 456-466.

Hodges, K., \& Gust, J. (1995). Measures of impairment for children and adolescents. Journal of Mental Health Administration, 22, 403-413.

Hodges, K., \& Kim, C. (2000). Psychometric study of the Child and Adolescent Functional Assessment Scale: Prediction of contact with the law and poor school attendance. Journal of Abnormal Child Psychology, 28, 287-297.

Hodges, K., \&Wong, M. M. (1996). Psychometric characteristics of a multidimensional measure to assess impairment: The Child and Adolescent Functional Assessment Scale. Journal of Child and Family Studies, 5, 445-467.

Holmbeck, G. N. (1997). Toward, terminological, conceptual, and statistical clarity in the study of mediators and moderators: Examples from the child-clinical and pediatric psychology literatures. Journal of Consulting and Clinical Psychology, 65, 599610. 
Howard, K. I., Lueger, R. J., \& Kolden, G. G. (1997). Measuring progress and outcome in the treatment of affective disorders. In H. H. Strupp, L. M. Horowitz, \& M. J. Lambert (Eds.), Measuring patient changes in mood, anxiety, and personality disorders: Toward a core battery (pp.263-282). Washington, DC: American Psychological Association.

Huber, P. J. (1967). The behavior of maximum likelihood estimates under nonstandard conditions. Proceedings of the Fifth Berkeley Symposium on Mathematical Statistics and Probability (pp. 221-233). Berkeley, CA: University of California Press.

Ialongo, N., Edelsohn, G., Werthamer-Larsson, L., Crockett, L., \& Kellam, S. (1995). The significance of self-reported anxious symptoms in first-grade children: Prediction to anxious symptoms and adaptive functioning in fifth grade. Journal of Child Psychology and Psychiatry, 36, 427-437.

Jaccard, J. (1998). Interaction effects in factorial analysis of variance. Newbury Park, CA: Sage.

Jaccard, J., \& Jacoby, J. (2010). Theory construction and model building skills: A practical guide for social scientists. New York: Guilford.

Jaccard, J., \& Turrisi, R. (2003). Interaction effects in multiple regression (2nd ed.). Thousand Oaks, CA: Sage.

Jaccard, J., Turrisi, R., \& Wan, C. (1990) Interaction effects in multiple regression. Newbury Park: Sage.

Jaccard, J., \& Wan, C. (1996). LISREL analyses of interaction effects in multiple regression. Newbury Park, CA: Sage.

Jacobson, N. S., Roberts, L. J., Berns, S. B., \& McGlinchey, J. B. (1999). Methods for defining and determining the clinical significance of treatment effects: Description, application, and alternatives. Journal of Consulting and Clinical Psychology, 67, 300-307.

Jensen, P. S., Rubio-Stipec, M., Canino, G., Bird, H. R., Dulcan, M. K., Schwab-Stone, M. E., \& Lahey, B. B. (1999). Parent and child contributions to diagnosis of mental disorder: Are both informants always necessary? Journal of the American Academy of Child \& Adolescent Psychiatry, 38, 1569-1579.

Johnston, C., \& Murray, C. (2003). Incremental Validity in the Psychological Assessment of Children and Adolescents. Psychological Assessment, 15, 496-507. 
Kazdin, A. E. (1994). Methodology, design, and evaluation in psychotherapy research. In A. E. Bergin \& S. L. Garfield (Eds.), Handbook of psychotherapy and behavior change (4th ed.) (pp. 19-71). New York: Wiley.

Kazdin, A. E. (1999). The meanings and measurement of clinical significance. Journal of Consulting and Clinical Psychology, 67, 332-339.

Kazdin, A. E. (2001). Progression of therapy research and clinical application of treatment require better understanding of the change process. Clinical Psychology: Science and Practice, 8, 143-151.

Kazdin, A. E. (2007). Mediators and mechanisms of change in psychotherapy research. Annual Review of Clinical Psychology, 3, 1-27.

Kazdin, A. E. (2008). Evidence-based treatment and practice: New opportunities to bridge clinical research and practice, enhance the knowledge base, and improve patient care. American Psychologist, 63, 146 -159.

Kendall, P. C. (1994). Treating anxiety disorders in children: Results of a randomized clinical trial. Journal of Consulting and Clinical Psychology, 62, 200-210.

Kendall , P. C., Hudson, J. L., Gosch, E., Flannery-Schroeder, E., \& Suveg, C. (2008). Cognitive-behavioral therapy for anxiety disordered youth: A randomized clinical trial evaluating child and family modalities. Journal of Consulting and Clinical Psychology, 76, 282-297.

Kenny, D. A. (1979). Correlation and causality. New York: John Wiley.

King, G., Honaker, J., Joseph, A., \& Scheve, K. (2001). Analyzing incomplete political science data: An alternative algorithm for multiple imputation. American Political Science Review, 95, 49-69.

Kramer, T. L., Phillips, S. D., Hargis, M. B., Miller, T. L., Burns, B. J., \& Robbins, J. M. (2004). Disagreement between parent and adolescent reports of functional impairment. Journal of Child Psychology and Psychiatry, 45, 248-259.

Kraemer, H. C., Wilson, G. T., Fairburn, C. G., \& Agras, W. S. (2002). Mediators and moderators of treatment effects in randomized clinical trials. Archives of General Psychiatry, 59, 877-884.

Kovacs, M., \& Devlin, B. (1998) Internalizing disorders in childhood. Journal of Child Psychology and Psychiatry, 39, 47- 63. 
Kurtines, W. M., \& Szapocznik, J. (1996). Structural family therapy in contexts of cultural diversity. In E. D. Hibbs \& P. S. Jensen (Eds.), Psychosocial treatment research of child and adolescent disorders. Washington, D. C.: American Psychological Association.

La Greca, A. M., Silverman, W. K., \& Lochman, J. E. (2009). Moving beyond efficacy and effectiveness in child and adolescent intervention research. Journal of Consulting and Clinical Psychology, 77, 373-82.

Lewinsohn, P. M., Seeley, J. R., Hibbard, J., Rohde, P., \& Sack, W. H. (1996). Crosssectional and prospective relationships between physical morbidities and depression in older adolescents. Journal of the American Academy of Child and Adolescent Psychiatry, 35, 1120-1129.

Little, R. J. A., \& Rubin, D. B. (1989). The analysis of social science data with missing values. Sociological Methods and Research, 18, 292-326.

MacKinnon, D. P., Lockwood, C. M., Hoffman, J. M., West, S. G., \& Sheets, V. (2002). A comparison of methods to test mediation and other intervening variable effects. Psychological Methods, 7, 83-104.

March, J. S., Parker, J., Sullivan, K., Stallings, P., \& Conners, K. (1997). The Multidimensional Anxiety Scale for Children (MASC): Factor structure, reliability and validity. Journal of the American Academy of Child and Adolescent Psychiatry, 36, 554-565.

March, J. S., \& Sullivan, K. (1999). Test-Retest Reliability of the Multidimensional Anxiety Scale for Children. Journal of Anxiety Disorders, 13, 349-358.

Marin, C. E. (2010). Parental Involvement and Group Cognitive Behavioral Treatment for Anxiety Disorders in Children and Adolescents: Treatment Specificity and Mediation Effects of Parent and Peer Variables (Doctoral dissertation, Florida International University). Retrieved from http://digitalcommons.fiu.edu/etd/256

Martens, M. P., \& Haase, R. F. (2006). Advanced Applications of Structural Equation Modeling in Counseling Psychology Research. The Counseling Psychologist, 34, 878-911.

Moreno, J., Silverman, W. K., Saavedra, L. M., \& Phares, V. (2008). Fathers' ratings in the assessment of their child's anxiety symptoms: A comparison to mothers' ratings and their associations with paternal symptomatology. Journal of Family Psychology, 22, 915-919.

Murray, L., Creswell, C., \& Cooper, P. J. (2009). The development of anxiety disorders in childhood: An integrative review. Psychological Medicine, 39, 1413-1423. 
Muthén, L. K.., \& Muthén, B. O. (2007). Mplus User's Guide (Sixth Edition). Los Angeles, CA: Muthén \& Muthén.

Nathan, P. E., \& Gorman, J. M. (2002). A guide to treatments that work (2nd ed.). London: Oxford University Press.

Ollendick, T. H. (1983). Reliability and validity of the revised Fear Survey Schedule for Children (FSSC-R). Behaviour Research and Therapy, 21, 685-692.

Panichelli-Mindel, S. M., Flannery-Schroeder, E., Kendall, P. C., \& Angelosante, A. G. (2005). Disclosure of distress among anxiety-disordered youth: Differences in treatment outcome. Journal of Anxiety Disorders, 19, 403-422.

Pela, O. A., \& Reynolds, C. R. (1982). Cross-cultural application of the revised-children's manifest anxiety scale: Normative and reliability data for Nigerian primary school children. Psychological Reports, 51, 1135-1138.

Rapee, R. M., Schniering, C. A., \& Hudson, J. L. (2009). Anxiety disorders during childhood and adolescence: Origins and treatment. Annual Review of Clinical Psychology, 5, 311-341.

Rausch, J. R., Maxwell, S. E., \& Kelley, K. (2003). Analytic methods for questions pertaining to a randomized pretest, post-treatment, follow up design. Journal of Clinical Child and Adolescent Psychology, 32, 467-486.

Reynolds, C. R., \& Richmond, B. O. (1978). What I think and feel: A revised measure of children's manifest anxiety. Journal of Abnormal Child Psychology, 6, 271-280.

Sanford, M. N., Offord, D. R., Boyle, M. H., Peace, A., \& Racine, Y. A. (1992). Ontario Child Health Study: Social and school impairments in children aged 6-16 years. Journal of the American Academy of Child and Adolescent Psychiatry, 31, 60-67.

Schorre, B., \& Vandvik, I. (2004). Global assessment of psychosocial functioning in child and adolescent psychiatry. A review of three unidimensional scales (CGAS, GAP, GAPD). European Child and Adolescent Psychiatry, 13, 273-286.

Shaffer, D., Gould, M. S., Brasic, J., Ambrosini, P., Fisher, P., Bird, H., \& Aluwahlia, S. (1983). A Children's Global Assessment Scale (CGAS). Archives of General Psychiatry, 40, 1228-1231.

Silverman, W. K., \& Albano, A. M. (1996). Anxiety Disorders Interview Schedule for Children-IV (Child and Parent Versions). San Antonio, TX: Psychological Corporation. 
Silverman, W. K., Cerny, J. A., \& Nelles, W. B. (1988). The familial influence in anxiety disorders: Studies on the offspring of patients with anxiety disorders. In B. B. Lahey \& A. E. Kazdin (Eds.), Advances in clinical child psychology, Vol.11 (pp. 223-248). New York: Plenum Press.

Silverman, W. K., \& Eisen, A. R. (1992). Age differences in the reliability of parent and child reports of child anxious symptomatology using a structured interview. Journal of American Academy of Child and Adolescent Psychiatry, 31, 117-124.

Silverman, W. K., \& Kurtines, W. M. (1996). Anxiety and phobic disorders: A pragmatic approach. New York: Plenum Press.

Silverman, W. K., Kurtines, W. M., Ginsburg, G. S., Weems, C. F., Lumpkin, P. W., \& Hicks-Carmichael, D. (1999). Treating anxiety disorders in children with group cognitive behavior therapy: A randomized clinical trial. Journal of Consulting and Clinical Psychology, 67, 995-1003.

Silverman, W. K., Kurtines, W. M., Jaccard, J., \& Pina, A. A. (2009). Directionality of change in youth anxiety treatment involving parents: An initial examination. Journal of Consulting and Clinical Psychology, 77, 474-485.

Silverman, W., \& Ollendick, T. (2005). Evidence-based assessment of anxiety and its disorders in children and adolescents. Journal of Clinical Child and Adolescent Psychology, 34, 380-411.

Silverman, W. K., Ortiz, C. D., Viswesvaran, C., Burns, B. J., Kolko, D. J., Putnam, F. W., \& Amaya-Jackson, L. (2008). Evidence based psychosocial treatments for children and adolescents exposed to traumatic events. Journal of Clinical Child and Adolescent Psychology, 37, 156-183.

Silverman, W. K., Pina, A. A., \& Viswesvaran, C. (2008). Evidence-based psychosocial treatments for phobic and anxiety disorders in children and adolescents. Journal of Clinical Child and Adolescent Psychology, 37, 105-130.

Silverman, W. K., Saavedra, L. M., \& Pina, A. A. (2001). Test-retest reliability of anxiety symptoms and diagnoses with the Anxiety Disorders Interview Schedule for DSM-IV: Child and Parent Versions. Journal of the American Academy of Child and Adolescent Psychiatry, 40, 937-944.

Satorra, A. (2000). Scaled and adjusted restricted tests in multi-sample analysis of moment structures. In R.D.H. Heijmans, D.S.G. Pollock, \& A. Satorra (Eds.), Innovations in multivariate statistical analysis. A Festschrift for Heinz Neudecker (pp. 233-247). London: Kluwer Academic Publishers. 
Steinhausen, H., (1987). Global assessment of child psychopathology. Journal of the American Academy of Child and Adolescent Psychiatry, 26, 203-206.

Steinhausen, H., \& Metzke. C. (2001). Global measures of impairment in children and adolescents: Results from a Swiss community survey. Australian and New Zealand Journal of Psychiatry, 35, 282-286.

Valderhaug, R., \& Ivarsson, T. (2005). Functional impairment in clinical samples of Norwegian and Swedish children and adolescents with Obsessive-Compulsive Disorder. European Child and Adolescent Psychiatry, 14, 164-173.

Vandvik, I. H. (1990). Mental health and psychological functioning in children with recent onset of rheumatic disease. Journal of Child Psychology and Psychiatry, 31, 961-971.

Üstun, B., \& Chatterji, S. (1997). Editorial: Measuring functioning and disability - A common framework. International Journal of Methods in Psychiatric Research, 7, 79-83.

Walkup, J. T., Albano, A. M., Piacentini, J., Birmaher, B., Compton, S. N., Joel T. Sherrill, J. T., ... Kendall, P. C. (2008). Cognitive Behavioral Therapy, Sertraline, or a Combination in Childhood Anxiety. The New England Journal of Medicine, 359, 2753-2766.

Weems, C. F., \& Costa, N. M. (2005). Developmental differences in the expression of childhood anxiety symptoms and fears. Journal of the American Academy of Child and Adolescent Psychiatry, 44, 656-663.

Wechsler, D. (2004). The Wechsler intelligence scale for children-fourth edition. London: Pearson Assessment.

Wells, K. B., Burnam, M.A., Rogers, W., Hays, R., \& Camp, P. (1992). The course of depression in adult outpatients: Results from the Medical Outcomes Study. Archives of General Psychiatry, 49, 788-794.

Wille, N., Bettge, S., Wittchen, H., \& Ravens-Sieberer, U. (2008). How impaired are children and adolescents by mental health problems? Results of the BELLA study. European Child and Adolescent Psychiatry, 1, 42-51.

White, H. (1980). A heteroskedasticity-consistent covariance matrix estimator and a direct test for heteroskedasticity. Econometrica, 48, 817-830.

Woodcock, R. W., McGrew, K. S., \& Mather, N. (2001). Woodcock-Johnson III Tests of Achievement. Itasca, IL: Riverside Publishing. 
Wothke, W. (2000). Longitudinal and multi-group modeling with missing data. In T. D. Little, K. U. Schnabel, \& J. Baumert (Eds.), Modeling longitudinal and multiple group data: Practical issues, applied approaches and specific examples (pp. 219240). Mahwah, NJ: Erlbaum.

Zahn-Waxler, C., Klimes-Dougan, B., \& Slattery, M. (2000). Internalizing disorders of childhood and adolescence: Progress and prospects for advances in understanding anxiety and depression. Development and Psychopathology, 12, 443-466. 
APPENDICES 
Table 1

Demographic and Diagnostic Information by Treatment Condition

\begin{tabular}{|c|c|c|c|c|c|c|c|c|}
\hline \multirow[b]{2}{*}{ Variable } & \multicolumn{4}{|c|}{$\operatorname{PCBT}(n=100)$} & \multicolumn{4}{|c|}{$\operatorname{GCBT}(n=83)$} \\
\hline & $n$ & $\%$ & $M$ & $S D$ & $n$ & $\%$ & $M$ & $S D$ \\
\hline Age (years) & & & 9.71 & 2.28 & & & 9.86 & 2.17 \\
\hline Gender (male) & 52 & 52 & & & 46 & 54 & & \\
\hline \multicolumn{9}{|l|}{ Target diagnosis } \\
\hline Separation anxiety & 40 & 40 & & & 34 & 41.0 & & \\
\hline Social phobia & 23 & 23 & & & 18 & 21.6 & & \\
\hline Specific phobia & 17 & 17 & & & 13 & 15.7 & & \\
\hline Generalized anxiety & 14 & 14 & & & 12 & 14.5 & & \\
\hline OCD & 3 & 3 & & & 2 & 2.4 & & \\
\hline PD w/ Agoraphobia & 1 & 1 & & & 2 & 2.4 & & \\
\hline PD w/out Agoraphobia & 1 & 1 & & & 1 & 1.2 & & \\
\hline Selective Mutism & 1 & 1 & & & 1 & 1.2 & & \\
\hline \multicolumn{9}{|l|}{ Ethnic background } \\
\hline Euro-American & 25 & 25 & & & 11 & 13.3 & & \\
\hline Hispanic/Latino & 69 & 69 & & & 68 & 81.9 & & \\
\hline African-American & 3 & 3 & & & 2 & 2.4 & & \\
\hline Other/not reported & 3 & 3 & & & 2 & 2.4 & & \\
\hline \multicolumn{9}{|l|}{ Annual income } \\
\hline$\$ 0-\$ 20,999$ & 10 & 10.9 & & & 15 & 19.7 & & \\
\hline$\$ 21,000-\$ 40,999$ & 19 & 20.7 & & & 18 & 23.7 & & \\
\hline$\$ 41,000-\$ 60,999$ & 16 & 17.4 & & & 12 & 15.8 & & \\
\hline$\$ 61,000-\$ 80,999$ & 12 & 13 & & & 9 & 11.8 & & \\
\hline$\$ 81,000-\$ 99,999$ & 11 & 12 & & & 8 & 10.5 & & \\
\hline$\$ 100,000-\$ 149,999$ & 13 & 14.1 & & & 9 & 11.8 & & \\
\hline$>\$ 150,000$ & 11 & 12 & & & 5 & 6.6 & & \\
\hline Not reported & 1 & 1.1 & & & 7 & 8.7 & & \\
\hline \multicolumn{9}{|l|}{ Marital Status } \\
\hline Married & 73 & 86.9 & & & 61 & 79.2 & & \\
\hline Divorced & 8 & 9.5 & & & 7 & 9.1 & & \\
\hline Single & 1 & 1.2 & & & 0 & 0.0 & & \\
\hline Separated & 2 & 2.4 & & & 5 & 6.5 & & \\
\hline Remarried & 0 & 0.0 & & & 2 & 2.6 & & \\
\hline
\end{tabular}

Note. Mother's Education $=$ Highest education mother attained; Father's Education $=$ Highest education father attained; PCBT $=$ Parent-involvement cognitive behavior treatment; GCBT = Group cognitive behavior treatment. 
Table 1 (Continued)

Demographic and Diagnostic Information by Treatment Condition

\begin{tabular}{|c|c|c|c|c|c|c|c|c|}
\hline \multirow[b]{2}{*}{ Variable } & \multicolumn{4}{|c|}{$\operatorname{PCBT}(n=100)$} & \multicolumn{4}{|c|}{$\operatorname{GCBT}(n=83)$} \\
\hline & $n$ & $\%$ & $M$ & $S D$ & $n$ & $\%$ & $M$ & $S D$ \\
\hline \multicolumn{9}{|l|}{ Unmarried living } \\
\hline $\mathrm{w} /$ partner & 0 & 0.0 & & & 2 & 2.6 & & \\
\hline Widowed & 0 & 0.0 & & & 0 & 0.0 & & \\
\hline Not reported & 1 & 1.1 & & & 3 & 3.8 & & \\
\hline \multicolumn{9}{|l|}{ Mother's Education } \\
\hline Grade school & 0 & 0.0 & & & 0 & 0.0 & & \\
\hline Some high school & 3 & 3.2 & & & 1 & 1.3 & & \\
\hline High school & 7 & 7.4 & & & 9 & 11.4 & & \\
\hline GED & 3 & 3.2 & & & 1 & 1.3 & & \\
\hline Some college & 13 & 13.7 & & & 19 & 24.1 & & \\
\hline College & 20 & 21.1 & & & 15 & 19.0 & & \\
\hline Bachelor's & 27 & 28.4 & & & 13 & 16.5 & & \\
\hline Master's & 12 & 12.6 & & & 9 & 11.4 & & \\
\hline Ph.D. & 3 & 3.2 & & & 2 & 2.5 & & \\
\hline Technical Degree & 3 & 3.2 & & & 8 & 10.1 & & \\
\hline Advanced Degree & 3 & 3.2 & & & 1 & 1.3 & & \\
\hline Other/Not Reported & 1 & 1.1 & & & 1 & 1.3 & & \\
\hline \multicolumn{9}{|l|}{ Father's Education } \\
\hline Some grade school & 1 & 1.1 & & & 1 & 1.3 & & \\
\hline Grade school & 2 & 2.2 & & & 1 & 1.3 & & \\
\hline Some high school & 3 & 3.2 & & & 5 & 6.3 & & \\
\hline High school & 6 & 6.5 & & & 7 & 8.9 & & \\
\hline GED & 2 & 2.2 & & & 3 & 3.8 & & \\
\hline Some college & 14 & 15.1 & & & 8 & 10.1 & & \\
\hline College & 19 & 20.4 & & & 16 & 20.3 & & \\
\hline Bachelor's & 21 & 22.6 & & & 15 & 19.0 & & \\
\hline Master's & 10 & 10.8 & & & 11 & 13.9 & & \\
\hline Ph.D. & 6 & 6.5 & & & 3 & 3.8 & & \\
\hline Technical Degree & 4 & 4.3 & & & 8 & 10.1 & & \\
\hline Advanced Degree & 4 & 4.3 & & & 1 & 1.3 & & \\
\hline Other/Not Reported & 1 & 1.1 & & & 0 & 0.0 & & \\
\hline
\end{tabular}

Note. Mother's Education $=$ Highest education mother attained; Father's Education = Highest education father attained; PCBT $=$ Parent-involvement cognitive behavior treatment; GCBT $=$ Group cognitive behavior treatment. 
Table 2

Mean (Standard Deviations) for Outcome and Mediator Measures, $N=183$

\begin{tabular}{llll}
\hline & Pretreatment & Posttreatment & Follow Up \\
\hline Outcome Measures & & & \\
CGAS & $55.34(5.31)$ & $76.09(13.03)$ & $79.23(13.83)$ \\
CSR (Combined) & $7.04(1.01)$ & $1.46(2.54)$ & $0.73(1.82)$ \\
CSR based on ADIS-C & $6.35(1.37)$ & $0.61(1.80)$ & $0.38(1.36)$ \\
CSR based on ADIS-P & $6.69(1.12)$ & $1.29(2.42)$ & $0.49(1.51)$ \\
Mediator Measures & $13.30(6.63)$ & & $6.91(6.54)$ \\
RCMAS & $57.03(21.95)$ & $7.45(6.37)$ & $44.89(42.52)$ \\
MASC & $12.82(5.64)$ & $41.28(19.48)$ & $6.28(5.30)$ \\
RCMAS/P & $8.25(5.75)$ & \\
\hline
\end{tabular}

Note. CGAS = Children's Global Assessment Scale; CSR = Clinician Severity Rating; ADIS-C = Anxiety Disorder Interview Schedule for DSM-IV/Child Version; ADIS-P= Anxiety Disorders Interview Schedule for DSM-IV/Parent Version; RCMAS = Revised Children's Manifest Anxiety; RCMAS/P = Revised Children's Manifest Anxiety/Parent Version; MASC $=$ Multidimensional Anxiety Scale for Children. 
Table 3

Mean Differences for Outcome and Youth Completed Measures

\begin{tabular}{lcccc}
\hline & Parameter & SE & $p$ Value & $95 \%$ CI \\
\hline CGAS at Post & 9.67 & 0.08 & $<.001$ & {$[5.18,14.15]$} \\
Low-High for RCMAS & 7.24 & 0.09 & .001 & {$[2.81,11.67]$} \\
Medium-High for RCMAS & 8.16 & 0.08 & .002 & {$[2.96,13.35]$} \\
Low-High for MASC & 8.72 & 0.08 & $<.001$ & {$[4.54,12.90]$} \\
Medium-High for MASC & & & & {$[-3.35,-0.98]$} \\
CSR at Post & -2.17 & 0.11 & $<.001$ & {$[-3.04,-0.70]$} \\
Low-High for RCMAS & -1.87 & 0.11 & .002 & .017 \\
Medium-High for RCMAS & -1.56 & 0.09 & {$[-2.84,-0.28]$} \\
Low-High for MASC & & & &
\end{tabular}

Note. CGAS = Children's Global Assessment Scale; CSR = Clinician Severity Rating; RCMAS = Revised Children's Manifest Anxiety; MASC = Multidimensional Anxiety Scale for Children; Post $=$ Posttreatment. 
Table 4

Mean Differences for Outcome and Parent Completed Measures

\begin{tabular}{lcccc}
\hline & Parameter & SE & $p$ Value & $95 \%$ CI \\
\hline CGAS at Post & 16.95 & 0.07 & $<.001$ & {$[12.46,21.45]$} \\
Low-High for RCMAS/P & 9.10 & 0.07 & $<.001$ & {$[5.18,13.06]$} \\
Medium-High for RCMAS/P & 7.86 & 0.06 & $<.001$ & {$[3.88,11.83]$} \\
Low-Medium for RCMAS/P & & & & {$[-3.17,-0.77]$} \\
CSR at Post & -1.97 & 0.09 & .001 & {$[-2.63,-0.42]$} \\
Low-High for RCMAS/P & -1.52 & 0.10 & .007 & {$[-1.39,-0.37]$} \\
Medium-High for RCMAS/P & & & & {$[-1.95,-0.30]$} \\
CSR at FU & -0.88 & 0.05 & .001 & .008 \\
Low-Medium for RCMAS/P & -1.12 & 0.08 & & \\
Low-High for RCMAS/P & & & & \\
\hline
\end{tabular}

Note. CGAS = Children's Global Assessment Scale; CSR = Clinician Severity Rating; RCMAS/P = Revised Children's Manifest Anxiety/Parent Version; Post = Posttreatment; FU = 12-month Follow Up. 
Table 5

Incremental Validity of the CGAS in relation to combined CSR

\begin{tabular}{|c|c|c|c|c|}
\hline & Adjusted $R^{2}$ & Adjusted $R^{2} w /$ CGAS & $R^{2}$ Change & $p$ Value \\
\hline \multicolumn{5}{|c|}{ Combined CSR at Post } \\
\hline Youth RCMAS & 0.17 & 0.40 & 0.23 & $<.001$ \\
\hline Youth MASC & 0.04 & 0.38 & 0.35 & $<.001$ \\
\hline Parent RCMAS/P & 0.14 & 0.38 & 0.23 & $<.001$ \\
\hline \multicolumn{5}{|c|}{ Combined CSR at FU } \\
\hline Youth RCMAS & 0.06 & 0.25 & 0.19 & $<.001$ \\
\hline Youth MASC & 0.04 & 0.25 & 0.22 & $<.001$ \\
\hline Parent RCMAS/P & 0.09 & 0.25 & 0.16 & $<.001$ \\
\hline
\end{tabular}

Note. CGAS = Children's Global Assessment Scale; Combined CSR = Clinician Severity Rating based on youths' and parents' views of impairment; RCMAS = Revised Children's Manifest Anxiety; RCMAS/P = Revised Children's Manifest Anxiety/Parent Version; MASC $=$ Multidimensional Anxiety Scale for Children; Post $=$ Posttreatment; FU = 12-month Follow Up. 
Table 6

Incremental Validity of the CGAS in relation to ADIS-C CSR

\begin{tabular}{|c|c|c|c|c|}
\hline & Adjusted $R^{2}$ & Adjusted $R^{2}$ w/ CGAS & $R^{2}$ Change & $p$ Value \\
\hline \multicolumn{5}{|c|}{ ADIS-C CSR at Post } \\
\hline Youth RCMAS & 0.15 & 0.20 & 0.06 & .001 \\
\hline Youth MASC & 0.09 & 0.14 & 0.06 & .001 \\
\hline \multicolumn{5}{|c|}{$A D I S-C C S R$ at $F U$} \\
\hline Youth RCMAS & 0.08 & 0.13 & 0.05 & .024 \\
\hline Youth MASC & 0.02 & 0.11 & 0.09 & .003 \\
\hline
\end{tabular}


Table 7

Incremental Validity of the CGAS in relation to ADIS-P CSR

\begin{tabular}{|c|c|c|c|c|}
\hline & Adjusted $R^{2}$ & Adjusted $R^{2}$ w/ CGAS & $R^{2}$ Change & $p$ Value \\
\hline \multicolumn{5}{|c|}{ ADIS-P CSR at Post } \\
\hline Parent RCMAS/P & 0.15 & 0.39 & 0.24 & $<.001$ \\
\hline \multicolumn{5}{|l|}{$A D I S-P C S R$ at $F U$} \\
\hline Parent RCMAS/P & 0.09 & 0.17 & 0.09 & .009 \\
\hline
\end{tabular}

Note. CGAS = Children's Global Assessment Scale; ADIS-P CSR = Clinician Severity Ratings of Impairment based on the Anxiety Disorders Interview Schedule for DSM-IV/Parent Version; RCMAS/P = Revised Children's Manifest Anxiety/Parent Version; MASC = Multidimensional Anxiety Scale for Children; w/ = with. 
Figure 1. Conceptual Mediation Model

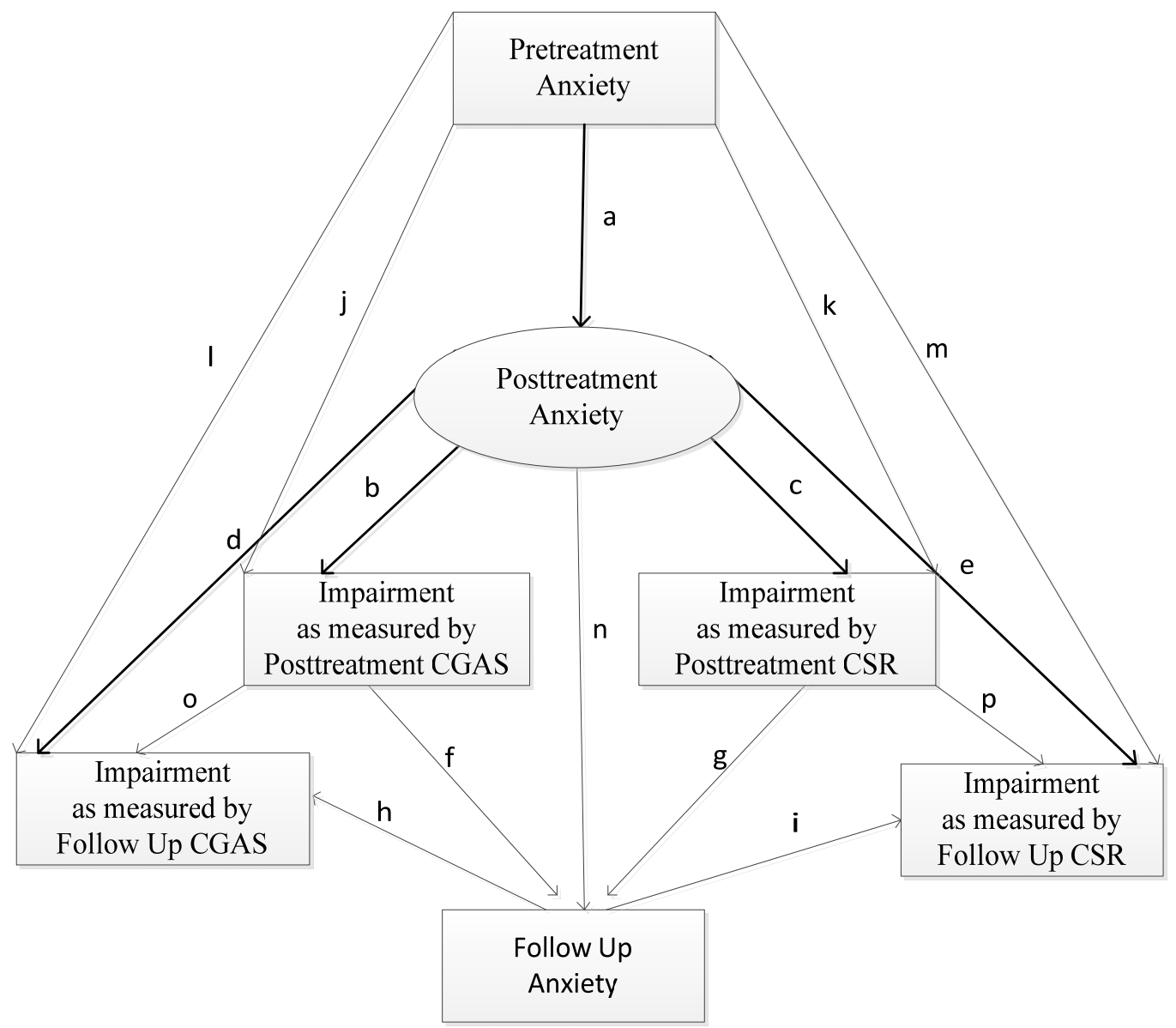


Figure 2. Youth Mediation Model with RCMAS

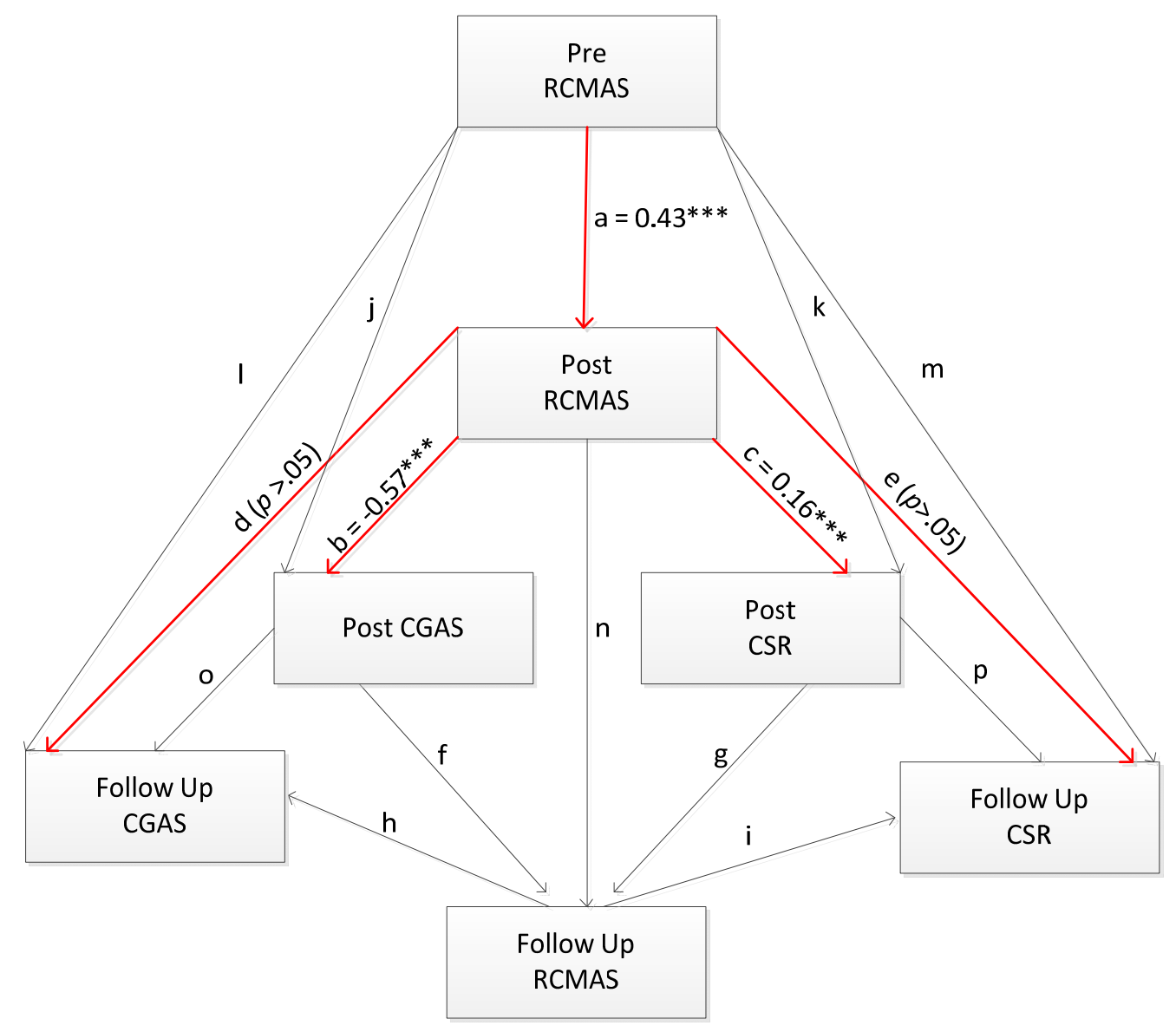

Note. RCMAS = Revised Children's Manifest Anxiety; CGAS = Children's Global Assessment Scale; CSR = Clinician Severity Rating.

$* * * p<.001$. 
Figure 3. Parent Mediation Model with RCMAS/P

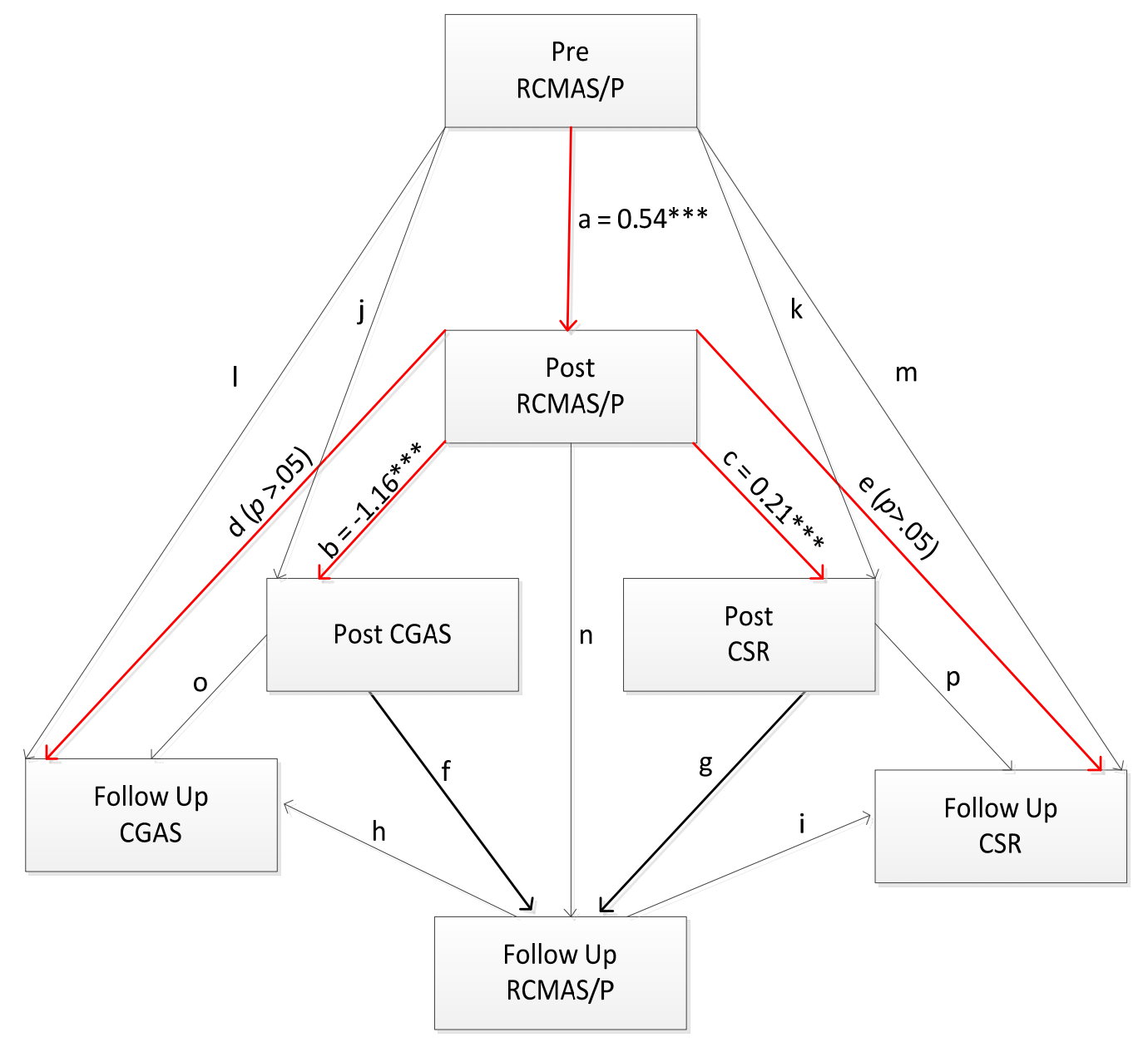

Note. RCMAS/P = Revised Children's Manifest Anxiety/Parent; CGAS = Children's Global Assessment Scale; CSR = Clinician Severity Rating. *** $p<.001$. 
Figure 4. Youth Mediation Model with Reverse Paths

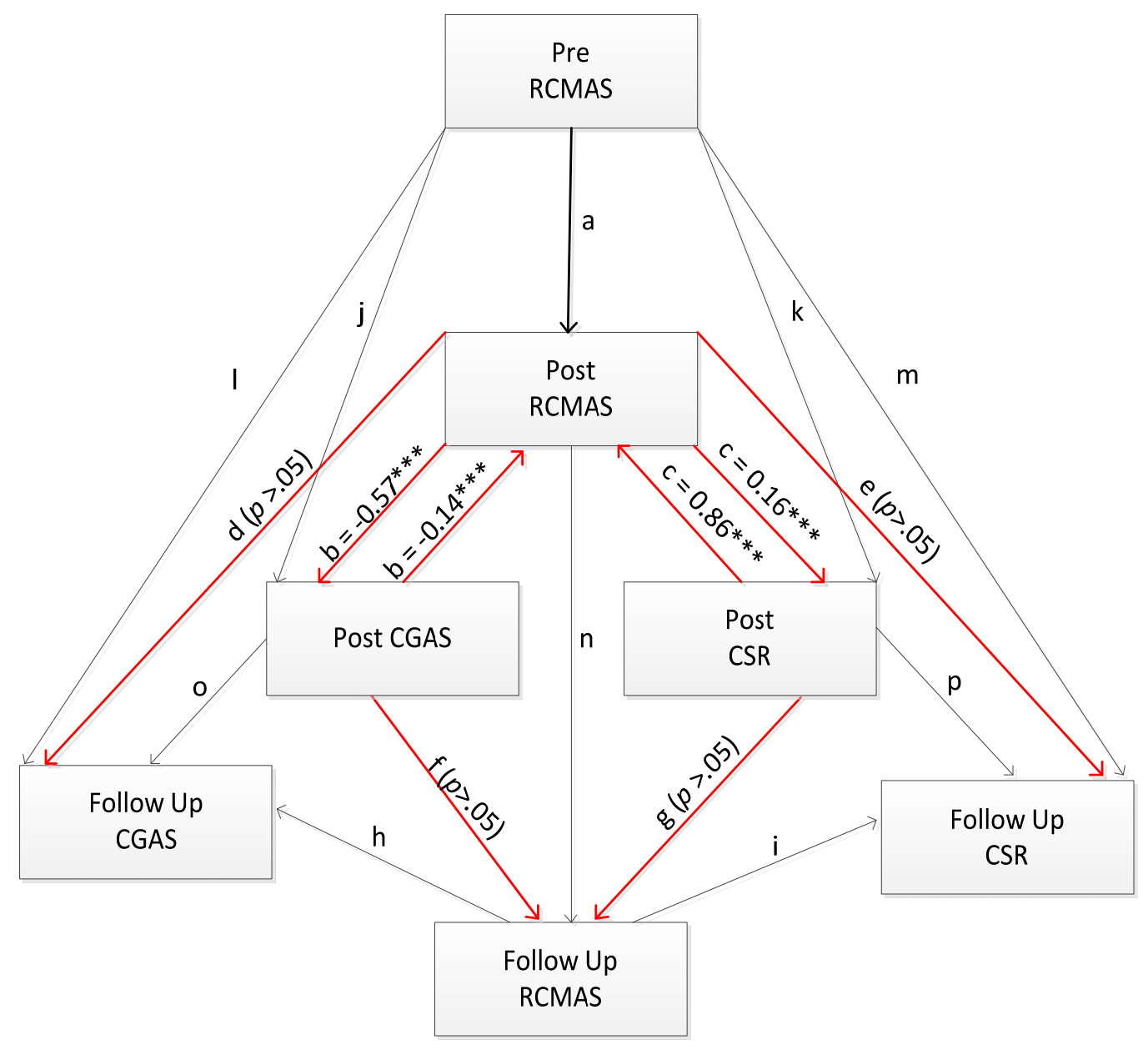

Note. RCMAS = Revised Children's Manifest Anxiety; CGAS = Children's Global Assessment Scale; CSR = Clinician Severity Rating. $* * * p<.001$. 
Figure 5. Parent Mediation Model with Reverse Paths

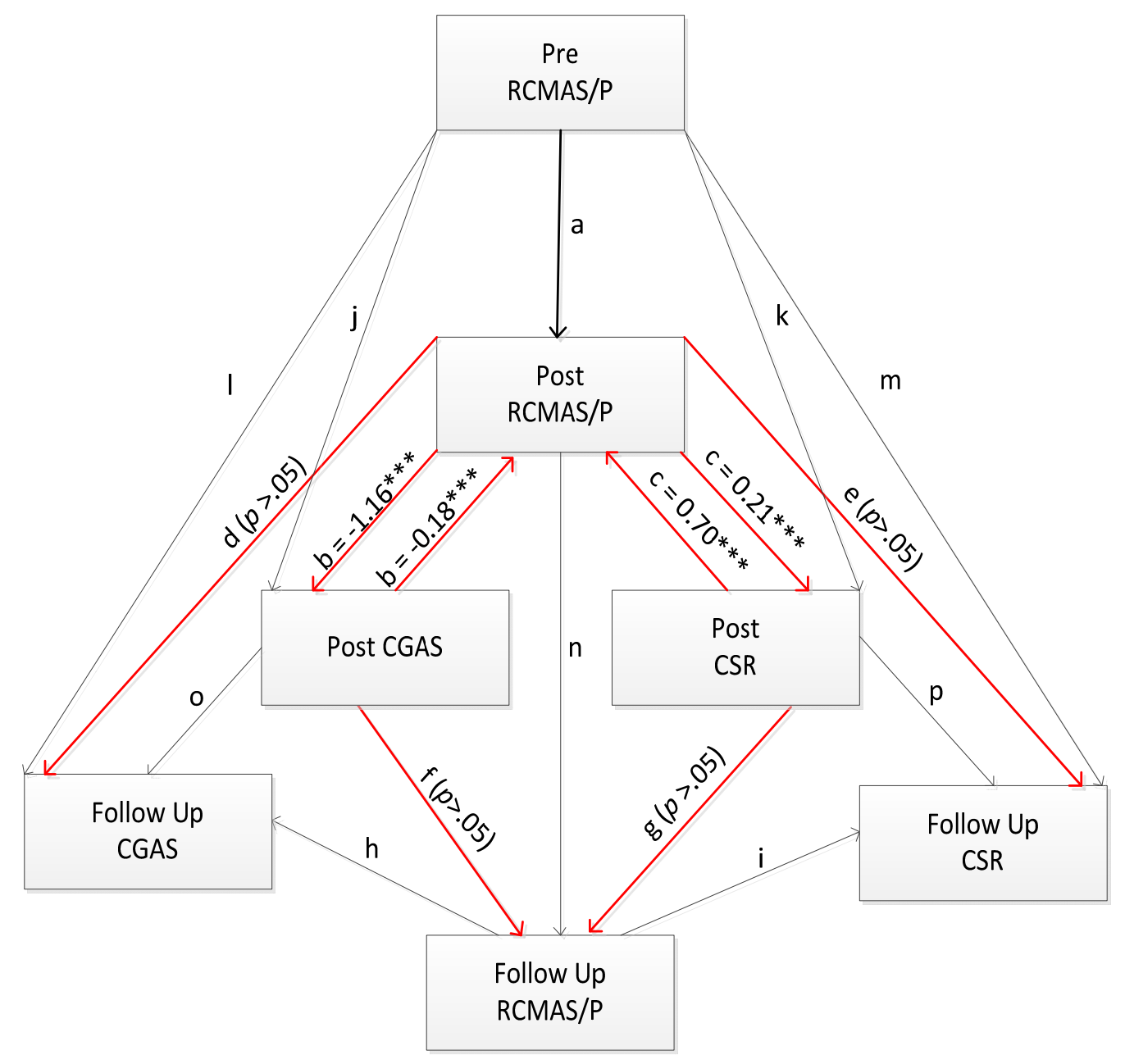

Note. $\mathrm{RCMAS} / \mathrm{P}=$ Revised Children's Manifest Anxiety/Parent; CGAS = Children's Global Assessment Scale; CSR = Clinician Severity Rating.

$* * * p<.001$. 
Figure 6. Youth Mediation Model with Product Term

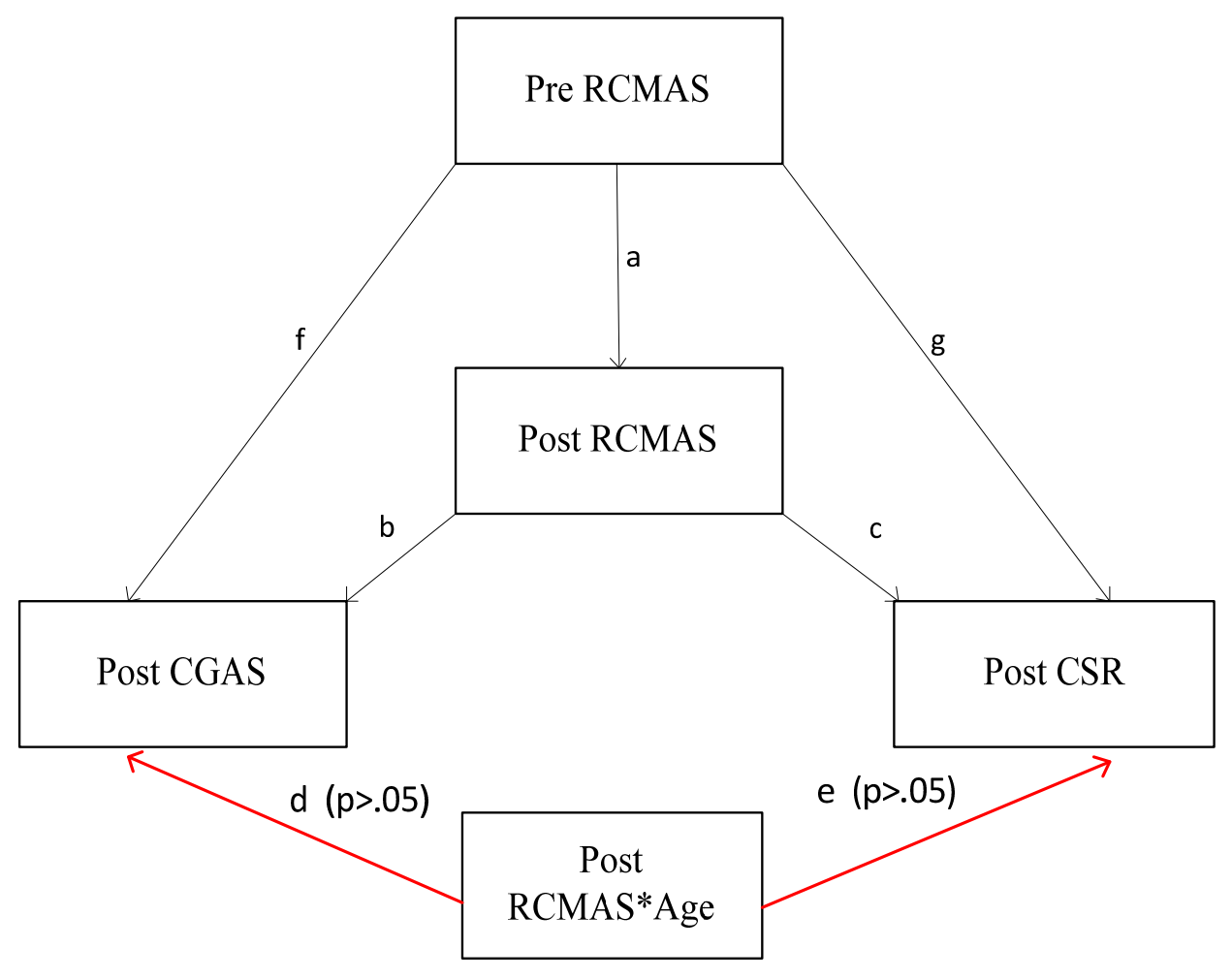

Note. RCMAS = Revised Children's Manifest Anxiety; CGAS = Children's Global Assessment Scale; $\mathrm{CSR}=$ Clinician Severity Rating; $\mathrm{RCMAS}^{*}$ Age = Interaction term between RCMAS and youth age. 
Figure 7. Parent Mediation Model with Product Term

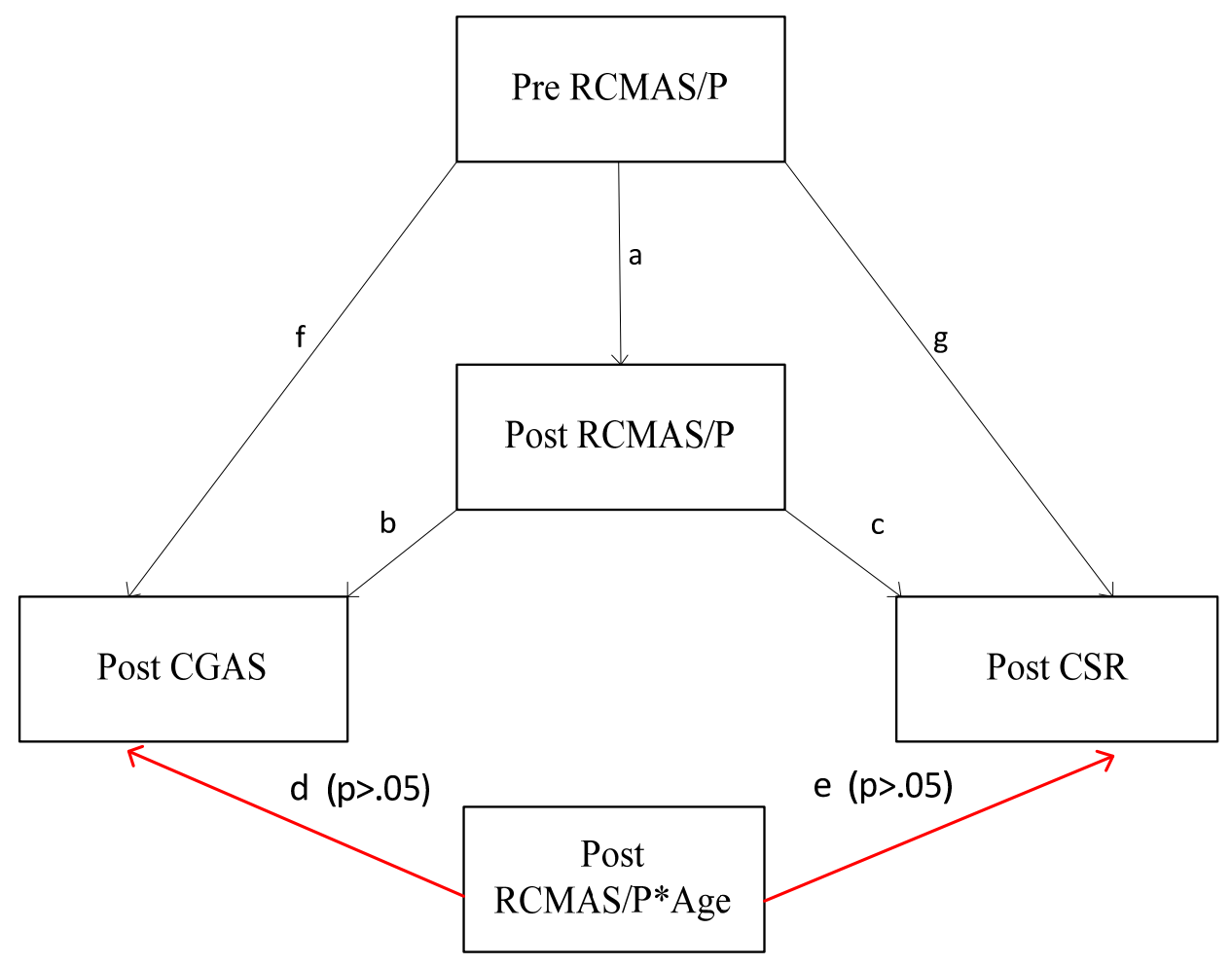

Note. $\mathrm{RCMAS} / \mathrm{P}=$ Revised Children's Manifest Anxiety/Parent Version; CGAS = Children's Global Assessment Scale; CSR = Clinician Severity Rating; RCMAS/P*Age $=$ Interaction term between RCMAS/P and youth age. 
Figure 8. Youth Mediation Model with ADIS-C CSR

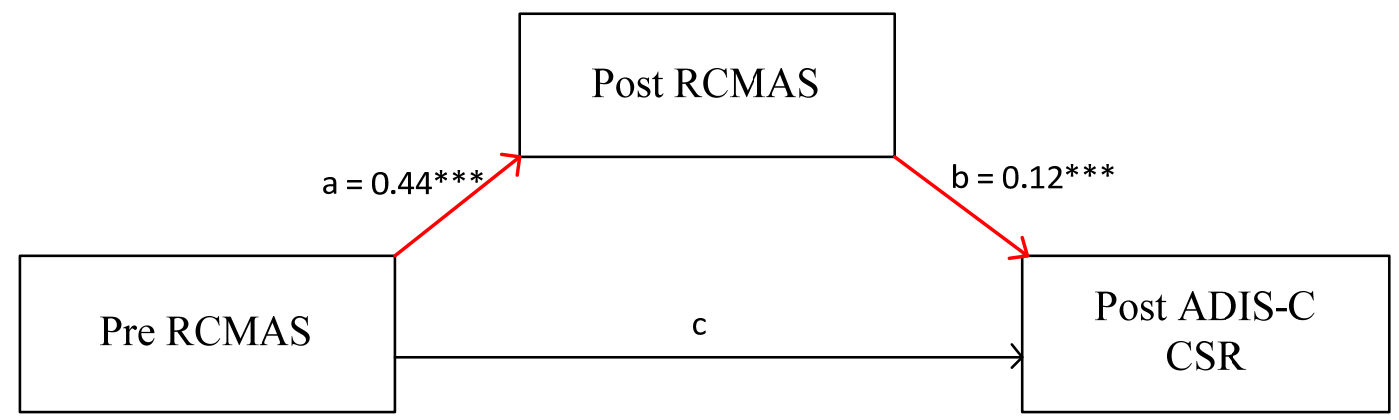

Note. RCMAS = Revised Children's Manifest Anxiety; ADIS-C CSR = Clinician Severity Rating of Impairment based on the Anxiety Disorders Interview Schedule for DSM-IV/Child Version.

$* * * p<.001$. 
Figure 9. Parent Mediation Model with ADIS-P CSR

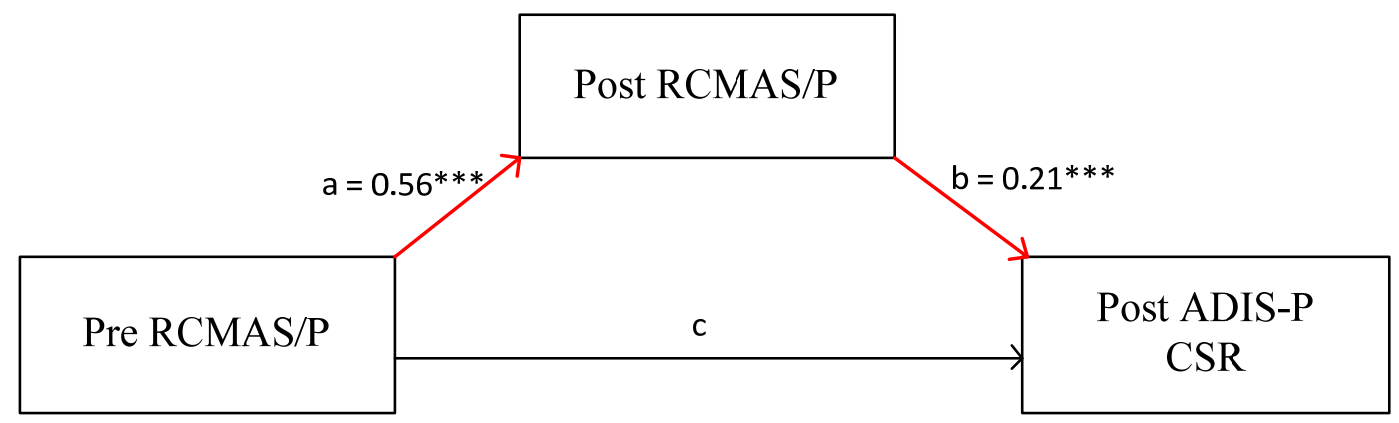

Note. RCMAS/P = Revised Children's Manifest Anxiety/Parent Version; ADIS-P CSR = Clinician Severity Ratings of Impairment based on the Anxiety Disorders Interview Schedule for DSM-IV/Parent Version.

$* * * \mathrm{p}<.001$. 


\section{Footnotes}

${ }^{1}$ Due to a significant amount of missing data at follow up (more than 10 percent on a given variable), the analyses using follow up ratings were pursued within Mplus 6 using the FIML methodology. Each estimated model in these analyses was just-identified and thus no fit indices are reported. Given that Mplus output does not provide the values of the $R^{2}$ change and its significance (i.e., the $p$ value), the $R^{2}$ change values were calculated manually, and its $p$ value was obtained from the SPSS output with the lesser $N$ (due to missing data).

${ }^{2}$ The total effect is a combination of the direct path from focal variable (e.g., Pre RCMAS) to outcome variable (e.g., Post CGAS and CSR) and the indirect effect as a result of the significant path from focal variable to the mediator (e.g., Post RCMAS).

${ }^{3}$ As discussed in Jaccard, Turrisi and Wan (1990) and Jaccard and Wan (1996), the product term approach is used when a proposed moderator is a continuous variable. Product terms are computed by multiplying a mediator variable (e.g., Post RCMAS) by a proposed moderating variable (e.g., youth age). The regression coefficient for the product term indicates the number of units that the slope for the regression of the outcome variable (e.g., Post CGAS and CSR) onto the mediator (e.g., Post RCMAS) changes, given a one-unit increase in the moderator (e.g., youth age). When including variables as product terms in the model, all continuous variables were mean centered by subtracting each participant's score from the mean of each variable. Mean centering is a good strategy to ease an interpretation of path coefficients and to counter any collinearity issues (Cohen \& Cohen, 1983; Jaccard \& Turrisi, 2003). 
${ }^{3}$ When a proposed moderator is a categorical variable, using the multiple group analysis approach is recommended (Baron \& Kenny, 1986). That is, a model in which the path coefficients were constrained to be equal across the groups of a given moderator (e.g., sex [i.e., boys and girls]) is tested first, followed by the analysis of an unconstrained model. The Satorra-Bentler method (Satorra, 2000) is pursued next to calculate the chi square difference between the constrained and unconstrained models. Its statistical significance was determined using a chi square critical values table, in which case an interaction effect for a given moderator is declared. 
VITA

\section{IRINA FREDERICKS}

2003

B.A., Psychology

Florida Atlantic University

Boca Raton, Florida

2008

M.S., Clinical Psychology

Barry University

Miami Shores, Florida

2008 to present

Doctoral Candidate, Developmental Psychology

Florida International University

Miami, Florida

\section{PUBLICATIONS AND PRESENTATIONS}

Marin, C. E., Motoca, L., M., Fredericks, I., Rey, Y., \& Silverman, W. K. (2011, November). The impact of academic transition and time of referral on symptoms severity and impairment in an anxious sample of youth. Poster presented at the annual meeting of the Association for Behavioral and Cognitive Therapies, Toronto, Canada.

Fredericks, I., Szuchman, L. T., \& Peterson, L. M. (2007, March). Against All Odds: Aggressive behavior and self-efficacy as the key factor for high-risk children's resilience. Paper presented at the $71^{\text {st }}$ Annual Meeting of the Florida Academy of Science.

University of South Florida, St. Petersburg, FL. Awarded by Florida Academy of Science as Outstanding Graduate Student Paper.

Fredericks, I., Szuchman, L.T., \& Peterson, L. M. (2006, March). Self-Efficacy as a Key Protective Factor in Resilience against the Development of Aggression. Poster presented at the Barry University $12^{\text {th }}$ Annual Psychology Student Research Forum. Barry University, Miami Shores, FL.

Beasley, S., Weekes-Shackelford, V. A., Fredericks, I., \& Shackelford, T. K. (2003, June). Filicide-suicide of stepparents and genetic parents. Paper presented at $15^{\text {th }}$ Annual Meeting of the Human Behavior and Evolution Society. University of Nebraska, Lincoln, NE.

Weekes-Shackelford, V. A., Fredericks, I., Beasley, S., \& Shackelford T. K. (2003, March). Wife-Killing Committed in the Context of a "Lovers Triangle." Poster presented at Florida Atlantic University Broward Psychology Fair. 